\title{
Backstepping PDE Design: A Convex Optimization Approach
}

\author{
Pedro Ascencio, Alessandro Astolfi, Fellow, IEEE, and Thomas Parisini, Fellow, IEEE
}

\begin{abstract}
Backstepping design for boundary linear PDE is formulated as a convex optimization problem. Some classes of parabolic PDEs and a first-order hyperbolic PDE are studied, with particular attention to non-strict feedback structures. Based on the compactness of the Volterra and Fredholm-type operators involved, their Kernels are approximated via polynomial functions. The resulting Kernel-PDEs are optimized using Sumof-Squares (SOS) decomposition and solved via semidefinite programming, with sufficient precision to guarantee the stability of the system in the $\mathcal{L}^{2}$-norm. This formulation allows optimizing extra degrees of freedom where the Kernel-PDEs are included as constraints. Uniqueness and invertibility of the Fredholm-type transformation are proved for polynomial Kernels in the space of continuous functions. The effectiveness and limitations of the approach proposed are illustrated by numerical solutions of some Kernel-PDEs.
\end{abstract}

Index Terms-Distributed Parameter Systems, Backstepping PDE design, Convex Optimization, Sum-of-Squares.

\section{INTRODUCTION}

$\mathbf{I}$ $\mathrm{N}$ the field of Distributed Parameter Systems (DPSs), continuous-time Backstepping for linear Partial Differential Equations (PDEs) is a well-established methodology in boundary control/observer design [1], [2], [3], [4], [5]. Its fundamental idea, the Volterra transformation [6], [7], traces back to the application of the method of Integral Operators for solving initial-boundary problems [8] derived from the boundary control of parabolic equations [9]. It stands out for its elegant and simple systematic methodology, which: (i) does not involve spatial discretization of the PDE model (see [10] for fundamental disadvantages of early lumping), (ii) carries out a collective treatment of the system modes instead of a finite analysis of them based on their spectral characteristics (see [11] and references therein), (iii) does not require to formulate the problem in abstract Hilbert spaces, apply semigroup theory, nor solve operator-valued equations (see [12], [13], [14] for extension of classical control theory to infinite-dimensional systems).

Backstepping design for PDEs involves two main problems: (i) the solution/well-posedness of the so-called Kernel-PDE

This work has been supported by the European Unions Horizon 2020 research and innovation programme under grant agreement No 739551 (KIOS CoE).

P. Ascencio is with the Warwick Manufacturing Group (WMG) at the University of Warwick, U.K., (p.ascencio.o@gmail.com).

A. Astolfi is with the Department of Electrical and Electronic Engineering, Imperial College London, London, U.K., and also with Dipartimento di Informatica, Sistemi e Produzione, Università di Roma "Tor Vergata", 00133 Rome, Italy (a.astolfi@ic.ac.uk).

T. Parisini is with the Department of Electrical and Electronic Engineering, Imperial College London, London, U.K., with the KIOS Research and Innovation Centre of Excellence, University of Cyprus, and also with the Dept. of Engineering and Architecture at the University of Trieste, Italy (t.parisini@gmail.com). and (ii) the invertibility of the integral transformation. This methodology has mostly been applied to systems known as "strict-feedback" systems on the basis of a Volterra-type transformation, invertibility of which is a well-know property [4], [15], [16]. It exploits the causal structure (causal in space [17]) leading to a kind of Kernel-PDE which is simple to solve in comparison with the operator Riccati equation derived from the linear quadratic regulator (LQR) approach [12], [18]. For some classes of these systems, the resulting Kernel-PDE can be reduced to a standard form, which allows obtaining a closed-form solution [19], [20]. For general cases a closedform analytic solution is hard to find and simple numeric methods cannot be applied directly [5].

A common methodology used to solve the Kernel-PDEs (as well as to prove their well-posedness), consists in transforming these differential formulations into integral equations to be solved via the Successive Approximation method. This way of solution has the objective to find a closed-form or provide a recursive computation of the integral Kernels [1]-[5], [6], [7], [19], [20], [21]. This kind of analysis is framed in the context of the Banach's contraction principle [22], tools also typically used to prove existence and uniqueness [7], [21], [23], [24]. Since, for strict-feedback systems, this type of analysis has provided a useful and simple numerical tool, somewhat reduced research efforts have been devoted to solve the Kernel-PDE in alternative ways.

Recently, Backstepping for PDEs has been implemented on systems with "non-strict" feedback structure on the basis of a Fredholm-type transformation, for parabolic [25], [26] as well as hyperbolic PDEs [27], [28]. These kind of systems arise from multiple sources. For instance, naturally, in dynamics with non-local terms involving the whole spatial domain, in PDE models [25] or in finite-dimensional systems with distributed delays [29]. Additionally, in design-oriented problems such as: control of coupled PDE-ODE (Ordinary Differential Equations) systems by under-actuated schemes [27], [28] (fewer actuators than spatial states [30]; it avoids an additional control action to cancel the non-strict feedback term [20]), or observer design for systems the output of which (sensing) comprises the states on the whole domain [31]. In these cases, a Volterra-type transformation cannot be used (at least directly) and the application of a Fredholm-type transformation leads to new and intricate mathematical problems (operator invertibility, Kernel solvability) [30]. For instance, from the application of the concepts of fixed point theory (Picard sequence of successive approximations [32]) arises some system parameters constraints to guaranty the uniqueness of the Kernel-PDE solution, and thus the convergence of an approximate solution and the invertibility of a Fredholm- 
type transformation [27], [28]. This is due to the necessary condition of contraction of the resulting operator (Kernels with small spectral radius), one of the main drawbacks of this methodology of analysis for addressing general cases [23]. On the contrary, if a particular Kernel structure is proposed, such as partially separable Kernels, a simplified analysis can be carried out based on the the method of separation of variables [26]. However, under this approach the invertibility of the integral transformation and the solvability of the resulting Kernel-PDE are limited to a specific class of coefficients of the system.

In this article a novel methodology to solve approximately the Kernel-PDEs for both Volterra and Fredholm-type operators is presented. The proposed methodology recasts the Kernel-PDE as a convex optimization problem which: (i) obtains approximate Kernel solutions with sufficient precision to guarantee the stability of the closed-loop system, (ii) is not subject to the spectral characteristics of the resulting approximate operators, and (iii) allows optimizing extra degrees of freedom where the Kernel-PDE is included as a constraint. Assuming the well-posedness of the Kernel-PDEs, the main objective of the proposed approach is to determine Kernels to guarantee the stability of the system, which allows relaxing the exact zero matching condition on the differential boundary problem. Polynomial Kernels are proposed as approximate solution of the resulting Kernel-PDEs and the minimization of the residual functions is addressed by means of polynomial optimization tools. In particular, a Sum-of-Squares (SOS) decomposition problem is formulated - equivalent to a convex optimization problem - readily implementable resorting to semidefinite programming tools. Moreover, existence and invertibility of the Fredholm-type transformation proposed in [26], [27] is proved in the Banach space of continuous functions and in the Hilbert space of square integrable realanalytic functions.

The paper is organized as follows. In Section II the essential background, definitions and technical results are briefly introduced. In Section III the problem of stabilization of parabolic PDE via the Volterra-type transformation is presented, while in Section IV the aim of determining an optimal target system is addressed. In Section V the stabilization of hyperbolic PDE via the Fredholm-type transformation is analysed. In Section VI numerical results for specific examples related to Sections III, IV and $\mathrm{V}$ are presented. Concluding remarks are given in Section VII.

A preliminary version of this paper has been published in [33]. Its application to the adaptive observer design problem has been presented in [34], [35].

\section{PRELIMINARIES}

\section{A. Notation}

$\mathcal{A}(\Omega), \mathcal{C}^{r}(\Omega)$ and $\mathcal{L}^{2}(\Omega)$ stand for the space of real-analytic functions, continuous functions with continuous first $r$ derivatives and square integrable functions on the domain $\Omega$, respectively. $\mathbb{I}, \mathbb{A}, \mathbb{V}$ and $\mathbb{F}$ denote the identity, integral, Volterra-type and Fredholm-type operator, respectively. $\mathbb{R}[\mathbf{x}]$ denotes the ring of real polynomials in $n$ variables $\mathbf{x}=\left[x_{1}, x_{2}, \ldots, x_{n}\right]^{T}$ and $\mathbb{P}[\mathbf{x}]=\left\{p \in \mathbb{R}[\mathbf{x}]: p(x) \geq 0, \forall x \in \mathbb{R}^{n}\right\}$ stands for the set of non-negative real polynomials. The notation $\mathbb{R}_{n, r}[\mathbf{x}]$ and $\mathbb{P}_{n, r}[\mathbf{x}]$ explicitly indicates polynomials in $n$ variables with degree at most $r$, whereas $\Sigma_{s}$ represents the subset of polynomials with Sum-of-Squares (SOS) decomposition. In particular $\mathbb{P}(\mathbf{K})$ represents the non-negative polynomials on the set $\mathbf{K}$. $\Phi_{r}=\left[1, x_{1}, \ldots, x_{n}, x_{1}^{2}, x_{1} x_{2}, \ldots, x_{n}^{r}\right]^{\top}$ is the standard vector basis of $\mathbb{R}_{n, r}[\mathbf{x}]$. Polynomials are expressed by multi-index notation: $p(\mathbf{x})=\sum_{j=0}^{z(r)-1} p_{j} \mathbf{x}^{\bar{\alpha}_{j}}=\left\langle\bar{p}, \Phi_{r}\right\rangle$, where $r \in \mathbb{N}$ is the polynomial degree, $z(r)=\left(\begin{array}{c}n+r \\ r\end{array}\right)$ is the number of polynomial coefficients $\bar{p}=\left[p_{0}, p_{1}, \ldots, p_{z(r)-1}\right]^{\top} \in \mathbb{R}^{z(r)}$, $\mathbf{x}^{\bar{\alpha}_{j}}=x_{1}^{\alpha_{j}^{1}} \cdots x_{n}^{\alpha_{j}^{n}}$ represents the $j$-th monomial with powers $\bar{\alpha}_{j}=\left[\alpha_{j}^{1}, \ldots, \alpha_{j}^{n}\right]$ such that $\left|\bar{\alpha}_{j}\right|=\sum_{k=1}^{n} \alpha_{j}^{k} \leq r, \alpha_{j}^{k} \in$ $\mathbb{N}$. An abstract form of this notation is given by $p(\mathbf{x})=$ $\sum_{\boldsymbol{\alpha} \in \mathbb{N}_{r}^{n}} p_{\boldsymbol{\alpha}} \mathbf{x}^{\boldsymbol{\alpha}}$ with powers $\boldsymbol{\alpha} \in \mathbb{N}_{r}^{n}$, where $\mathbb{N}_{r}^{n}=\left\{\bar{\alpha}_{j} \in\right.$ $\left.\mathbb{N}^{n} ;\left|\bar{\alpha}_{j}^{r}\right| \leq r, \forall j=0, \ldots, z(r)-1\right\}=\left\{\bar{\alpha}_{0}, \ldots, \bar{\alpha}_{z(r)-1}\right\} . \mathbb{S}_{+}^{m}$ denotes the set of symmetric positive semidefinite matrices of dimension $m \times m$.

\section{B. Integral Compact Operators}

Linear differential equations, ODEs and PDEs (boundaryvalue or initial-value problems), can be transformed into linear integral equations, the operators of which are frequently bounded or compact (completely continuous) [23], [36], [37]. In fact, every linear integral operator $\mathbb{A}: \mathcal{X} \rightarrow \mathcal{X}$ :

$$
\mathbb{A}[u(\cdot)](\mathbf{x}):=\int_{\Omega \subset \mathbb{R}^{n}} K(\mathbf{x}, \mathbf{y}) u(\mathbf{y}) d \mathbf{y}
$$

with continuous Kernel or weakly singular Kernel $K$, is compact on the Banach space of continuous functions $(\mathcal{X}=$ $\left.\left(\mathcal{C}(\Omega ; \mathbb{R}),\|\cdot\|_{\infty}\right)\right)$ and on the Hilbert space of square integrable functions $\left(\mathcal{X}=\left(\mathcal{L}^{2}(\Omega ; \mathbb{R}),\langle\cdot, \cdot\rangle_{\mathcal{L}^{2}}\right)\right)$. Likewise, for square integrable Kernels, $\mathbb{A}$ is compact on this Hilbert space [23], [38]. This is the case for the integral operators derived from the Kernel-PDEs in the Backstepping PDE design, as pointed out in [2] (page 19, footnote 2), where the Kernel is bounded and twice continuously differentiable.

In infinite-dimensional spaces bijectivity is a sufficient and necessary condition for (bounded) invertibility of bounded linear operators [16], [37]. For linear equations of second kind; namely

$$
(\mathbb{I}-\mathbb{A})[u(\cdot)](\mathbf{x})=w(\mathbf{x}),
$$

two approaches are commonly carried out to determine whether there exists a bounded inverse. The first method is framed in the context of the "Banach contraction principle" [22], [32], based on the so-called Neumann series, for bounded operator with small spectral radius $(\|A\|<1)$ [23]. This is the essential tool in the standard Backstepping PDE methodology, which relies on the inherent contraction property of the Volterra operator [39], guaranteeing the uniform convergence of the Successive Approximation method [6], [7], [21], [23]. The second method is a re-statement of the celebrated Fredholm Alternative theorem [23], [37], based on the compactness property of $\mathbb{A}$, which is the core of the proposed approach. In this case the existence of a unique trivial solution $u=0$ of the homogeneous equation $u-\mathbb{A} u=0$ implies invertibility and thus the uniqueness of solutions. 
Compact operators resemble the behaviour of operators in finite-dimensional spaces. In most of the traditional Banach spaces and for all Hilbert spaces, every compact operator is a limit of finite rank operators [38]. For continuous Kernels, a simple option for establishing this sequence are polynomials, which are a particular class of degenerate Kernels [23]. For instance if $K_{N}:=\sum_{j=0}^{N} k_{j} x^{\alpha_{j}} y^{\beta_{j}}$ then $\mathbb{A}_{K_{N}}[u(\cdot)](x)=$ $\int_{\Omega} K_{N}(x, y) u(y) d y$ is a polynomial in the span of $\left\{x^{\alpha_{j}}\right\}_{j=0}^{N}$ so that $\mathbb{A}_{K_{N}}$ is a finite rank operator and hence compact [38]. Moreover, since $K \in \mathcal{C}(\Omega \times \Omega)(\Omega=[0,1])$, based on the Weierstrass approximation theorem [40], there exists a sequence of polynomials $\left\{K_{N}\right\}_{N=0}^{\infty}$ such that:

$$
\left\|\mathbb{A}_{K}-\mathbb{A}_{K_{N}}\right\|=\sup _{\|u\| \leq 1}\left\|\mathbb{A}_{K-K_{N}} u\right\| \leq\left\|K-K_{N}\right\|_{\infty} \underset{N \rightarrow \infty}{\rightarrow} 0
$$

with $k_{j} \in \mathbb{R}, \alpha_{j} \in \mathbb{N}$ and $\beta_{j} \in \mathbb{N}$ for $j=0, \ldots, N$. Equivalent results can be obtained for square integrable Kernels [6], [41].

\section{Polynomial Optimization: Sum-of-Squares}

In general, the global polynomial optimization problem:

$$
\mathbf{P}:\left\{p^{*}=\inf _{\mathbf{x} \in \mathbf{K}} p(\mathbf{x}) \Leftrightarrow\left\{\begin{aligned}
p^{*}= & \sup \gamma \\
\text { subj. to: } & p(\mathbf{x})-\gamma \geq 0, \\
& \mathbf{x} \in \mathbf{K}
\end{aligned}\right.\right.
$$

where $\mathbf{K}:=\left\{\mathbf{x} \in \mathbb{R}^{n} ; g_{j}(\mathbf{x}) \geq 0, j=1, \ldots, m\right\}, g_{j} \in$ $\mathbb{R}[\mathbf{x}]$ and $p \in \mathbb{R}[\mathbf{x}]$, is NP-hard ${ }^{1}$. However, the problem $\mathbf{P}$ can be efficiently approximated by a hierarchy of convex (semidefinite) relaxations $\left(\mathbf{P}_{d} ; p_{d}^{*}\right)$, with $p_{d}^{*}$ global optimum for $(p(\mathbf{x})-\gamma) \in \mathbb{P}_{n, 2 d}[\mathbf{x}]$, using SOS representations for nonnegative polynomials [44], [45], [42] or the theory of Moments [46], [47], [48].

Theorem 1. ([42], [47]) Let $\Phi_{r}$ be the standard vector basis of $\mathbb{R}[\mathbf{x}]$ with $z(r)=\left(\begin{array}{c}n+r \\ r\end{array}\right)$ monomials in $\mathbf{x}$ with degree $\leq r . A$ multivariate polynomial $p \in \mathbb{R}[\mathbf{x}]$ is $\operatorname{SOS}\left(p \in \Sigma_{s} \subset \mathbb{P}_{n, 2 d}[\mathbf{x}]\right)$ if and only if there exists a matrix $Q \in \mathbb{S}_{+}^{z(d)}$ satisfying $p(\mathbf{x})=$ $\Phi_{d}^{T} Q \Phi_{d}, r=2 d$.

For $\mathbf{K}$ compact basic semi-algebraic set, the so-called Positivstellensatz of Schmüdgen [49] and Putinar [50] allows formulating the hierarchy of semidefinite relaxations of (4) as:

$\mathbf{P}_{d}:\left\{\begin{aligned} p_{d}^{*}= & \sup _{Q_{k}} \gamma \\ \text { subj. to : } & (p(\mathbf{x})-\gamma-\sum_{k=1}^{N} \overbrace{\Phi_{i_{k}}^{\top}(\mathbf{x}) Q_{k} \Phi_{i_{k}}(\mathbf{x})}^{s_{k}(\mathbf{x})} H_{k}(\mathbf{x})) \in \Sigma_{s} \\ & Q_{k} \in \mathbb{S}_{+}^{z\left(i_{k}\right)}, i_{k}=d-d_{k}, \forall k=1, \ldots, N,\end{aligned}\right.$

based on the existence of $s_{k} \in \Sigma_{s}$, where ${ }^{2} d_{k}=\left\lceil\operatorname{deg}\left(H_{k}\right) / 2\right\rceil$ and $\max _{k}\left(\operatorname{deg}(p), \operatorname{deg}\left(H_{k}\right)\right) \leq 2 d ; N=2^{m}$ and $H_{k}=$

\footnotetext{
${ }^{1}$ The right hand side of (4) sets forth the dual formulation of $\mathbf{P}$, which cannot be solved in polynomial time for quartic or higher degree polynomials [42]. However, its non-negative constraints can be approximated, amongst others [43], via SOS, providing a convex formulation with computational tractable solution via semidefinite programming (interior point method, smallmedium size problems).

${ }^{2}$ The function $\lceil a\rceil$, commonly referred to as the ceil function, rounds to the nearest integer greater than or equal to $a$.
}

$\prod_{k \in J} g_{k}$ for $J \subseteq\{1, \ldots, m\}$ if Schmüdgen's Positivstellensatz is considered; $N=m$ and $H_{k}=g_{k}$ for Putinar's Positivstellensatz ${ }^{3}$. Moreover, the approximate optimal solutions $p_{k}^{*}$ form a monotone nondecreasing sequence $\left(p_{d}^{*} \leq p_{d+1}^{*}\right)$ such that $p_{d}^{*} \rightarrow p^{*}$ as $d \rightarrow \infty$ [51].

\section{Convex Formulation of Differential BVPs}

Let $u=u(\mathbf{x}) \in \mathcal{C}^{w}(\Omega)$ be the solution of a linear PDEBoundary Value Problem (BVP):

$$
\mathbf{P}:\left\{\begin{aligned}
\mathbb{L}[u(\cdot)](\mathbf{x}) & =f(\mathbf{x}), \forall \mathbf{x} \in \stackrel{\Omega}{\Omega}, \\
\left.\mathbb{B}_{i}[u(\cdot)](\mathbf{x})\right|_{\mathbf{x} \in \partial \Omega_{i}} & =\mathbf{u}_{i}, \quad i=1, \ldots, r \in \mathbb{N},
\end{aligned}\right.
$$

in a compact domain $\Omega \subset \mathbb{R}^{n}$, where $\mathbb{L}$ and $\mathbb{B}_{i}$ are linear differential operators with polynomials terms, $f \in \mathbb{R}[\mathbf{x}], \partial \Omega \supseteq$ $\bigcup_{i=1}^{r} \partial \Omega_{i}$ represents the boundary of $\Omega=\Omega \cup \partial \Omega$, with $\Omega$ the interior of $\Omega$, and $\mathbf{u}_{i} \in \mathbb{R}$ is the value of $\mathbb{B}_{i}[u]$ on the boundary $\partial \Omega_{i}$. Based on the Weierstrass approximation theorem [52], the solution $u$ can be uniformly approximated by polynomials with theoretical arbitrary precision. Let

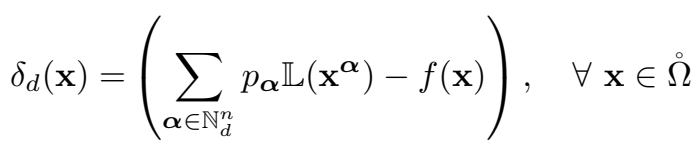

be the residual function due to the polynomial approximation $u(\mathbf{x}) \approx \mathbf{p}_{d}(\mathbf{x})=\sum_{\boldsymbol{\alpha} \in \mathbb{N}_{d}^{n}} p_{\boldsymbol{\alpha}} \mathbf{x}^{\boldsymbol{\alpha}} \in \mathbb{R}[\mathbf{x}]$ in (6), of degree $d$ in $n$ variables $\mathbf{x}=\left[x_{1}, \ldots, x_{n}\right]^{\top} \in \mathbb{R}^{n}$ and coefficients $\boldsymbol{p}=\left[p_{\alpha_{0}}, \ldots, p_{\alpha_{z(n, d)-1}}\right]^{\top} \in \mathbb{R}^{z(n, d)}$.

The main idea to solve (6) as a Polynomial Optimization Problem is based on a notable result from Real Algebraic Geometry: the Positivstellensatz [42]. Peculiarly, this result of positivity certification does not depend on the characteristics of the polynomials involved in the problem. On the contrary, this result only relies on the kind of algebraic representation of the domain $\Omega$. Thus, if $\Omega$ can be described by

$$
\Omega=\left\{\mathbf{x} \in \mathbb{R}^{n} ; g_{1}(\mathbf{x}) \geq 0, \ldots, g_{m}(\mathbf{x}) \geq 0\right\},
$$

with $m \in \mathbb{N}$ and $g_{j} \in \mathbb{R}[\mathbf{x}], \forall j=1, \ldots, m$, and this description is a compact basic semi-algebraic set, based on the representation theorems of Schmüdgen or Putinar, the nonnegative function $h(\mathbf{x})= \pm \delta_{d}(\mathbf{x}) \mp \gamma$, for some $\gamma \geq 0$, can be formulated as:

$$
\left( \pm \delta_{d}(\mathbf{x}) \mp \gamma-\sum_{J \neq 0} s_{J}(\mathbf{x}) G_{J}(\mathbf{x})\right) \in \Sigma_{s}, \quad s_{J} \in \Sigma_{s},
$$

$\forall \mathbf{x} \in \Omega$, where $G_{J}$ denotes a particular combination of polynomial constraints $g_{j}$ 's in accordance with the Schmüdgen or Putinar representation selected. Thus, (9) is a SOS decomposition problem equivalent to a convex optimization problem, numerically implementable via semidefinite programming [42], [48].

\section{Minimax Approximation}

Due to the axiom of completeness and its consequence on the generalized Min-Max theorem [53], the residual function

\footnotetext{
${ }^{3}$ Putinar's refinement requires that the quadratic module generated by $g_{1}, \ldots, g_{m}$ be Archimedean, which is not very restrictive [48]
} 
(7) is bounded by $\underline{\delta} \leq \delta_{d}(\mathbf{x}) \leq \bar{\delta}$, where $\underline{\delta}$ is the minimum and $\bar{\delta}$ is the maximum of $\delta_{d}$ on $\Omega$ compact domain. In addition, the exact solution of (6), i.e. $\delta_{d}=0$ in (7), can be approximated using the simple idea of imposing $\underline{\delta} \rightarrow 0$ and $\bar{\delta} \rightarrow 0\left(\delta_{d} \rightarrow 0\right.$ in $\left.\Omega\right)$ on the extreme values of $\delta_{d}$. Intuitively, to achieve this objective, the optimization problem $\min \left\{\max _{x \in \Omega}\{|\bar{\delta}|,|\underline{\delta}|\}<\gamma\right\}$ can be formulated. This can be seen as the standard Uniform Best approximation approach used to approximate the zero function by $\delta_{d}$ in terms of $\mathcal{L}^{\infty}$-norm (uniform error) in $\Omega$, a scheme also denominated Minimax approximation [54].

Similarly, a Least Squares approximation, namely $\min _{p_{\alpha}} \int_{\Omega} \delta_{d}^{2}(\mathbf{x}) d \mathbf{x}$, can be carried out based on polynomial matrix inequalities and the Schur's complement.

\section{E. Definitions and Technical Results}

For the representation of bivariate polynomial Kernels $P(x, y)=\sum_{k=0}^{z(d)-1} p_{k} x^{\alpha_{k}} y^{\beta_{k}}$ of degree $d \in \mathbb{N}$, coefficients $p_{k} \in \mathbb{R}$ and powers $\alpha_{k} \in \mathbb{N}, \beta_{k} \in \mathbb{N}$, the following standard basis of monomials is considered:

$$
\begin{gathered}
\Phi_{d}:=\left\{1, x, y, x^{2}, x y, y^{2}, x^{3}, x^{2} y, x y^{2}, y^{3}, \ldots\right. \\
\left.\ldots, x^{d}, x^{d-1} y, \ldots, x y^{d-1}, y^{d}\right\},
\end{gathered}
$$

with $z(d)=(d+1)(d+2) / 2$ terms ordered according to the monomials $\Phi_{d}(k)=x^{j-k} y^{k}, \forall k=0, \ldots, j$ and $j=0, \ldots, d$.

Lemma 1. The Backstepping design for 1-dimensional PDEs with Volterra or Fredholm-type transformation involves the domains: $\Omega:=\{0 \leq x \leq 1\}, \Omega_{L}:=\{0 \leq x \leq 1,0 \leq y \leq x\}$, $\Omega_{U}:=\{0 \leq x \leq 1, x \leq y \leq 1\}$ [1], [28], which can be formulated as:

$$
\begin{aligned}
\Omega & \equiv\left\{x \in \mathbb{R} ; g_{1}(x)=x(1-x) \geq 0\right\}, \\
\Omega_{L} & \equiv\left\{(x, y) \in \mathbb{R}^{2} ; g_{1}(x) \geq 0, g_{2}(x, y)=y(x-y) \geq 0\right\}, \quad(11) \\
\Omega_{U} & \equiv\left\{(x, y) \in \mathbb{R}^{2} ; g_{1}(x) \geq 0, g_{3}(x, y)=(y-x)(1-y) \geq 0\right\} .
\end{aligned}
$$

These domain representations are compact basic semialgebraic sets and their associated quadratic modules are Archimedean.

Proof: See the Appendix.

\section{Parabolic PDE and the Volterra Operator}

\section{A. Problem Setting}

In this section a class of parabolic PDEs with strict-feedback structure and spatially varying reactivity is considered [1], [2]:

$$
\begin{aligned}
u_{t}(x, t) & =\epsilon u_{x x}(x, t)+\lambda(x) u(x, t) \\
u(0, t) & =0, \quad u(1, t)=U(t),
\end{aligned}
$$

where $u(x, t)=u_{0}(x) \in \mathcal{C}(\Omega ; \mathbb{R})$ is the initial condition. The objective is to find a control action $U=U(t)$ so that the origin of (12) is finite-time stable in the topology of the $\mathcal{L}^{2}$-norm. For this class of systems the Backstepping PDE methodology proposes a Volterra-type transformation (here referred to as Volterra operator):

$$
\begin{aligned}
w(x, t) & =u(x, t)-\int_{0}^{x} K(x, y) u(y, t) d y \\
& =\left(\mathbb{I}-\mathbb{V}_{K}\right)[u(\cdot, t)](x)
\end{aligned}
$$

where $\mathbb{I}$ is the identity operator and $\mathbb{V}_{K}: \mathcal{C}(\Omega ; \mathbb{R}) \rightarrow \mathcal{C}(\Omega ; \mathbb{R})$, to transform the system (12) into the target stable system:

$$
\begin{aligned}
w_{t}(x, t) & =\epsilon w_{x x}(x, t)-c(x) w(x, t), \\
w(0, t) & =0, \quad w(1, t)=0,
\end{aligned}
$$

where $c(x) / \epsilon>-\pi^{2} / 4, \forall x \in \Omega$, with a boundary feedback control determined by $U(t)=\int_{0}^{1} K(1, y) u(y, t) d y$. Following the standard Backstepping PDE design procedure detailed in [1], the transformed system (12) takes the form:

$$
\begin{aligned}
& w_{t}(x, t)-\epsilon w_{x x}(x, t)+c(x) w(x, t)=\underbrace{\epsilon K(x, 0)}_{\delta_{0}(x)} u_{x}(0, t)+ \\
& \underbrace{\left((\lambda(x)+c(x))+2 \epsilon \frac{d}{d x} K(x, x)\right)}_{\delta_{1}(x)} u(x, t)+ \\
& \int_{0}^{x} \underbrace{\left(\epsilon K_{x x}(x, y)-\epsilon K_{y y}(x, y)-(\lambda(y)+c(y)) K(x, y)\right)}_{\delta_{2}(x, y)} u(y, t) d y,
\end{aligned}
$$

so that the target system (14) is achievable if the continuous bounded Kernel $K=K(x, y)$ satisfies the so-called KernelPDE:

$$
\delta_{2}(x, y)=0, \quad \delta_{1}(x)=0, \quad \delta_{0}(x)=0,
$$

$\forall(x, y) \in \Omega_{L}$ where the $\delta_{i}$ are defined in (15). In this article $\delta_{i}$ are denominated as "residual functions". This linear hyperbolic PDE (Klein-Gordon-type) is well-posed and, for constant reactivity terms $\lambda=\lambda_{0}$ and $c=c_{0}$, it can be solved in closed-form [1], [2], [19]:

$$
K^{\star}(x, y)=-\bar{\lambda} y I_{1}(\sqrt{\Theta}) / \sqrt{\Theta},
$$

in terms of the first-order modified Bessel function $I_{1}$ with $\bar{\lambda}=\left(\lambda_{0}+c_{0}\right) / \epsilon$ and $\Theta=\bar{\lambda}\left(x^{2}-y^{2}\right)$.

\section{B. Kernel-PDE as a Convex Optimization Problem}

\section{Proposition 1. Let}

$$
u(x, t)=w(x, t)+\int_{0}^{x} L(x, y) w(y, t) d y
$$

be the inverse transformation of (13) [1], [15] and:

$$
L(x, y)=\breve{L}(x, y)-\sigma, \breve{L}(x, y) \geq 0, \sigma \geq 0
$$

a positive decomposition of $L$ in the triangular domain $\Omega_{L}$. Let $m_{0,0}$ be the 0 -order moment of $\breve{L}$ in accordance with

$$
m_{i, j}:=\int_{0}^{1} \int_{0}^{x} x^{i} y^{j} \breve{L}(x, y) d y d x .
$$

Let $c(x) \geq \underline{c}>-\epsilon \pi^{2} / 4, \forall x \in \Omega, \overline{\delta_{1}}=\max _{x \in \Omega}\left|\delta_{1}(x)\right|$, $\overline{\delta_{2}}=\max _{(x, y) \in \Omega_{L}}\left|\delta_{2}(x, y)\right|$ and $\delta_{0}(x)=0$, the transformed system (15) is exponentially stable in the $\mathcal{L}^{2}$-norm topology if the residual functions satisfy:

$$
\overline{\delta_{1}}+\overline{\delta_{2}} \leq \min \left\{\frac{\epsilon \theta\left(\frac{\pi^{2}}{4}+1\right)+(\underline{c}-\epsilon)}{(1+\sigma)}, \frac{\epsilon(1-\theta)}{\left(m_{0,0}+\frac{\sigma+1 / 2}{4}\right)}\right\},
$$

for some scalar $0 \leq \theta \leq 1$.

Proof: See the Appendix.

Motivated by the result of Proposition 1, which sets forth a margin of clearance in the stability of this transformed 
system, a relaxation of the exact zero matching condition for the residual functions $\delta_{1}$ and $\delta_{2}$ can be considered. It allows formulating an approximate solution for the Kernel-PDE (16).

Proposition 2. Let $N(x, y)=\sum_{k=0}^{z(d)-1} n_{k} x^{\alpha_{k}} y^{\beta_{k}}$ be $a$ polynomial approximation of $K$ of arbitrary even degree $d>d_{r}=\max \left\{d_{\lambda}, d_{c}\right\} \in \mathbb{N}$ in accordance with (10). Let $\delta_{1}=\delta_{1}(x)$ and $\delta_{2}=\delta_{2}(x, y)$ be the resulting residual functions according to (15) with polynomial degrees $d_{1}=d$ and $d_{2}=2\left\lceil\frac{d+d_{r}+2}{2}\right\rceil$, respectively; let $\rho_{1}, \rho_{2}, \bar{\rho}_{1}$, and $\bar{\rho}_{2}$ be lower and upper bounds of these functions in $\Omega_{L}$; $\gamma_{j} \geq 0, j=1, \ldots, 4$. For reactivity terms $\lambda=\lambda(x)$ and $c=c(x)$ described by polynomial functions of degree $d_{\lambda}$ and $d_{c}$, respectively, the Kernel-PDE (16) can be formulated as the convex optimization problem:

$$
\underset{\gamma_{j}, N, s_{j}}{\operatorname{minimize}:} \quad \gamma_{1}+\gamma_{2}+\gamma_{3}+\gamma_{4}
$$

subject to:

$$
\begin{aligned}
& \left(\delta_{1}(x)-\underline{\rho}_{1}-s_{1}(x) g_{1}(x)\right) \in \Sigma_{s} \\
& \left(\bar{\rho}_{1}-\delta_{1}(x)-s_{2}(x) g_{1}(x)\right) \in \Sigma_{s} \\
& \left(\delta_{2}(x, y)-\underline{\rho}_{2}-\left[s_{3}(x, y) s_{4}(x, y)\right] g_{L}(x, y)\right) \in \Sigma_{s}, \\
& \left(\bar{\rho}_{2}-\delta_{2}(x, y)-\left[s_{5}(x, y) s_{6}(x, y)\right] g_{L}(x, y)\right) \in \Sigma_{s}, \\
& s_{j} \in \Sigma_{s}, \forall j=1, \ldots, 6, \\
& {\left[\begin{array}{ll}
\gamma_{1} & \underline{\rho}_{1} \\
\underline{\rho}_{1} & \gamma_{1}
\end{array}\right] \succeq 0, \quad\left[\begin{array}{cc}
\gamma_{2} & \bar{\rho}_{1} \\
\bar{\rho}_{1} & \gamma_{2}
\end{array}\right] \succeq 0} \\
& {\left[\begin{array}{ll}
\gamma_{3} & \underline{\rho}_{2} \\
\rho_{2} & \gamma_{3}
\end{array}\right] \succeq 0, \quad\left[\begin{array}{cc}
\gamma_{4} & \bar{\rho}_{2} \\
\bar{\rho}_{2} & \gamma_{4}
\end{array}\right] \succeq 0} \\
& \epsilon N_{x x}(x, y)-\epsilon N_{y y}(x, y)-(\lambda(y)+c(y)) N(x, y)=\delta_{2}(x, y)
\end{aligned}
$$$$
(\lambda(x)+c(x))+2 \epsilon N_{x}(x, x)=\delta_{1}(x),
$$$$
\delta_{0}(x)=N(x, 0)=0 \text {, }
$$

$\forall(x, y) \in \Omega_{L}$, for some polynomials $s_{1}, s_{2}$ of degree $d_{1}-$ $2, s_{3}, s_{4}, s_{5}, s_{6}$ of degree $d_{2}-2$ and $g_{L}=\left[g_{1}, g_{2}\right]^{\top}$. The optimal minimal bounds for the residual functions are: $\overline{\delta_{1}}=$ $\max \left\{\gamma_{1}, \gamma_{2}\right\}$ and $\overline{\delta_{2}}=\max \left\{\gamma_{3}, \gamma_{4}\right\}$.

Proof: Since $N$ is a polynomial approximation of $K$, as which is indicated above, the residual functions in (15) have a polynomial structure determined by (30)-(32). In addition, the quadratic module associated to the representation of the domains $\Omega$ and $\Omega_{L}$ are Archimedean (see Lemma 1). Based on Putinar's Positivstellensatz [51], [48], via the SOS decomposition (23)-(27), the unknown extreme values of $\delta_{1}$ and $\delta_{2}\left(\rho_{1}, \bar{\rho}_{1}, \rho_{2}, \bar{\rho}_{2}\right)$ can be determined via a polynomial optimization problem, which is convex in terms of polynomial coefficients and solved via semidefinite programming [42]. The absolute value of these upper and lower bounds are given by means of (28)-(29), so that the linear cost function (22) yields $\delta_{1} \rightarrow 0, \delta_{2} \rightarrow 0$ in $\Omega$ and $\Omega_{L}$, respectively.

\section{Parabolic PDE and Target System OPTIMIZATION}

\section{A. Problem Setting}

This section addresses the problem of optimizing the reactivity coefficient $c=c(x)$ in the target system (14) to achieve the smallest $\mathcal{L}^{2}$-norm of the Kernel acting as control action in (12) designed via the Volterra transformation (13), namely

$\underset{c=c(x), x \in \Omega}{\operatorname{minimize}:} \quad \int_{0}^{1} K^{2}(1, y) d y$

subject to:

$$
\begin{aligned}
& K(x, 0)=0, \quad 2 \epsilon \frac{d K}{d x}(x, x)=-(\lambda(x)+c(x)), \\
& \epsilon K_{x x}(x, y)-\epsilon K_{y y}(x, y)-(\lambda(y)+c(y)) K(x, y)=0,
\end{aligned}
$$

$\forall(x, y) \in \Omega_{L}$. Due to the quadratic term $K^{2}$ in the cost function (33) and the product $c(y) K(x, y)$ in (35), this optimization problem is nonlinear, and in general, non-convex.

\section{B. Convex Formulation of the Target Optimization Problem}

The complexity of the problem (33)-(35) can be circumvented if the following relation is considered. Let $P \in \mathcal{C}$ be a Kernel such that:

$$
\begin{aligned}
& K(x, y)=\frac{d K}{d y}(y, y)+2 P(x, y) \Rightarrow \\
& K(x, y) \frac{d K}{d y}(y, y)=\left(\frac{d K}{d y}(y, y)\right)^{2}+2 P(x, y) \frac{d K}{d y}(y, y)+ \\
& P^{2}(x, y)-P^{2}(x, y) \\
& =\left(\left[\frac{d K}{d y}(y, y)+2 P(x, y)\right]-P(x, y)\right)^{2}-P^{2}(x, y) \\
& =\underbrace{(K(x, y)-P(x, y))^{2}}_{h_{1}(x, y)}-\underbrace{P^{2}(x, y)}_{h_{2}(x, y)} .
\end{aligned}
$$

Thus, since from (34) $(\lambda(y)+c(y)) K(x, y)=$ $-2 \epsilon \frac{d K}{d y}(y, y) K(x, y)$, the product $c(y) K(x, y)$ can be considered via the term $\frac{d K}{d y}(y, y) K(x, y)=h_{1}(x, y)-h_{2}(x, y)$ as in (37). In addition, the functions

$$
\begin{aligned}
h_{1}(x, y) & =(K(x, y)-P(x, y))^{2}, h_{2}(x, y)=P^{2}(x, y), \\
f(y) & =K^{2}(1, y),
\end{aligned}
$$

in (37) and (33), respectively, can be obtained as solution of an optimization problem based on the following proposition.

Proposition 3. Let $q \in \mathcal{C}(\Omega): \Omega \rightarrow[0, \infty)$ and $\psi \in \mathcal{C}(\Omega)$ : $\Omega \rightarrow \mathbb{R}$. The function $q=\psi^{2}, \forall \mathbf{x} \in \Omega$, is the optimal solution of the optimization problem:

$$
\begin{aligned}
\underset{q \in C(\Omega)}{\operatorname{minimize}:} & \int_{\Omega} q(\mathbf{x}) d \Omega \\
\text { subject to: } & {\left[\begin{array}{cc}
q(\mathbf{x}) & \psi(\mathbf{x}) \\
\psi(\mathbf{x}) & 1
\end{array}\right] \succeq 0 . }
\end{aligned}
$$

Proof: On the basis of the Schur's complement, the inequality (40) is satisfied if and only if $\Delta(\mathbf{x})=q(\mathbf{x})-\psi^{2}(\mathbf{x}) \geq 0, \forall \mathbf{x} \in \Omega$. Thus, $\min _{q}\left\{\int_{\Omega} q(\mathbf{x}) d \Omega\right\}=\min _{q}\left\{\int_{\Omega}\left(\Delta(\mathbf{x})+\psi^{2}(\mathbf{x})\right) d \Omega\right\}=$ $\min _{q}\left\{\int_{\Omega} \Delta(\mathbf{x}) d \Omega\right\}+\int_{\Omega} \psi^{2}(\mathbf{x}) d \Omega=0+\int_{\Omega} \psi^{2}(\mathbf{x}) d \Omega$. Therefore, since $q \geq 0$, this equality is verified if and only if $q=\psi^{2}, \forall \mathbf{x} \in \Omega$.

Based on the non-negativity of the functions in (38), if polynomial functions are considered, the problem (33)-(35) can be approximately solved via SOS decomposition and convex optimization. 
Proposition 4. Let $N \in \mathbb{R}_{2, d}[(x, y)]$ be a polynomial approximation of $K$ of arbitrary even degree $d>\max \left\{d_{\lambda}, d_{c}\right\} \in \mathbb{N}$ in accordance with (10). Let $\delta_{j}$ be residual functions, with $\delta_{0}, \delta_{1}$, and $\delta_{2}$ as in (15) and $\delta_{3}=N(x, y)-\left(\frac{d N}{d y}(y, y)+2 P(x, y)\right)$ according to (36) for some Kernel $P \in \mathbb{R}_{2, d}[(x, y)]$, where $\underline{\rho}_{j}$ and $\bar{\rho}_{j}$ are their lower and upper bounds, respectively. Let $\underline{\rho}_{c}$ be the lower bound of $c=c(x)$. For reactivity coefficients $\bar{\lambda}^{c}=\lambda(x)$ and $c=c(x)$ described by polynomials of degree $d_{\lambda}$ and $d_{c}$, respectively, (33)-(35) can be formulated as the convex optimization problem:

$$
\begin{array}{r}
\underset{\gamma_{j}, N, P, c(x), h_{j}, f, s_{j}}{\operatorname{minimiz}} \sigma_{0} \int_{0}^{1} f(x) d x+\sigma_{1} \int_{0}^{1} \int_{0}^{x} h_{1}(x, y) d y d y+ \\
\sigma_{2} \int_{0}^{1} \int_{0}^{x} h_{2}(x, y) d y d x+\sum_{j=1}^{3} \gamma_{j}
\end{array}
$$

subject to:

$$
\begin{aligned}
& \left(\delta_{1}(x)-\underline{\rho}_{1}-s_{11}(x) g_{1}(x)\right) \in \Sigma_{s}, \\
& \left(\bar{\rho}_{1}-\delta_{1}(x)-s_{12}(x) g_{1}(x)\right) \in \Sigma_{s}, \\
& \left(\delta_{j}(x, y)-\underline{\rho}_{j}-\left[s_{j 1}(x, y) s_{j 2}(x, y)\right] g_{L}(x, y)\right) \in \Sigma_{s} \\
& \left(\bar{\rho}_{j}-\delta_{j}(x, y)-\left[s_{j 3}(x, y) s_{j 4}(x, y)\right] g_{L}(x, y)\right) \in \Sigma_{s}, \\
& \forall j=2,3,
\end{aligned}
$$$$
\left[\begin{array}{cc}
\gamma_{j} & \underline{\rho}_{j} \\
\underline{\rho}_{j} & \gamma_{j}
\end{array}\right] \succeq 0, \quad\left[\begin{array}{cc}
\gamma_{j} & \bar{\rho}_{j} \\
\bar{\rho}_{j} & \gamma_{j}
\end{array}\right] \succeq 0, \forall j=1,2,3,
$$

$\gamma_{j} \geq 0, \gamma_{j} \leq \Gamma_{0}, \forall j=1,2,3$,

$\delta_{0}(x)=N(x, 0)=0$,

$\delta_{1}(x)=(\lambda(x)+c(x))+2 \epsilon N_{x}(x, x)$,

$\delta_{2}(x, y)=N_{x x}(x, y)-N_{y y}(x, y)+2\left(h_{1}(x, y)-h_{2}(x, y)\right)$

$\delta_{3}(x, y)=N(x, y)-\left(\frac{d N}{d y}(y, y)+2 P(x, y)\right)$,

$$
\left[\begin{array}{cc}
h_{1}(x, y)-s_{41}(x, y) g_{1}(x) & N(x, y)-P(x, y) \\
N(x, y)-P(x, y) & 1-s_{42}(x, y) g_{2}(x)
\end{array}\right] \in \Sigma_{s}^{2 \times 2},
$$$$
\left[\begin{array}{cc}
h_{2}(x, y)-s_{51}(x, y) g_{1}(x) & P(x, y) \\
P(x, y) & 1-s_{52}(x, y) g_{2}(x)
\end{array}\right] \in \Sigma_{s}^{2 \times 2},
$$$$
\left[\begin{array}{cc}
f(y)-s_{6}(y) g_{1}(y) & N(1, y) \\
N(1, y) & 1
\end{array}\right] \in \Sigma_{s}^{2 \times 2},
$$$$
\left(c(x)-\underline{\rho}_{c}-s_{7}(x) g_{1}(x)\right) \in \Sigma_{s}, \quad \underline{\rho}_{c} \geq \Gamma_{1},
$$

$s_{11}, s_{12}, s_{j k} \in \Sigma_{s}, \forall j=2,3, k=1, \ldots, 4$,

$s_{i l}, s_{6}, s_{7} \in \Sigma_{s}, \forall i=4,5, l=1,2$,

$\forall(x, y) \in \Omega_{L}$, for some polynomials $s_{11}, s_{12}$ of degree $d-2$, $s_{2 k}$ of degree $2 d-2, s_{3 k}$ of degree $d-2, s_{i l}, s_{6}$ and $s_{7}$ of degree $2 d-2, \forall k=1, \ldots, 4, i=4,5, l=1,2$ and $g_{L}=\left[g_{1}, g_{2}\right]^{\top}$; where $\Gamma_{0}$ is selected to achieve a required precision in the approximate solution of the Kernel-PDE (34)(35), $\Gamma_{1}>-\epsilon \pi^{2} / 4, \forall x \in \Omega$, is selected according to the threshold of stability for (14), $\sigma_{0}>0, \sigma_{1}>0$ and $\sigma_{2}>0$ are weigh factors in the cost function, and $h_{1} \in \mathbb{R}_{2,2 d}[(x, y)]$, $h_{2} \in \mathbb{R}_{2,2 d}[(x, y)]$ and $f \in \mathbb{R}_{1,2 d}[(x, y)]$.

Proof: The convex formulation of (42)-(50) follows similar arguments as the ones given in the proof of Proposition
2. The residual $\delta_{2}$ in (50) is an equivalent formulation of (35) by means of substituting the boundary condition (34) into (35) and using the relation described in (37). The residual $\delta_{3}$ in (51) sets forth the condition (36), which is a necessary condition to use the relation (37). Based on Proposition 3, the matrix inequalities

$$
\begin{aligned}
A_{1} & =\left[\begin{array}{cc}
h_{1}(x, y) & N(x, y)-P(x, y) \\
N(x, y)-P(x, y) & 1
\end{array}\right] \succeq 0, \\
A_{2} & =\left[\begin{array}{cc}
h_{2}(x, y) & P(x, y) \\
P(x, y) & 1
\end{array}\right] \succeq 0, \\
A_{3} & =\left[\begin{array}{cc}
f(y) & N(1, y) \\
N(1, y) & 1
\end{array}\right] \succeq 0,
\end{aligned}
$$

$\forall(x, y) \in \Omega_{L}$, allow finding the functions $h_{1}, h_{2}$ and $f$ given in (38) via the objective defined in (41). The semi-definite conditions (57)-(59) are made computationally tractable via the matrix-polynomial version of Putinar's Positivstellensatz [48], [55], based on the representation of $\Omega_{L}$ as in Lemma 1, namely, for some $\rho>0, A(x, y) \succ \rho I \succ 0, \forall(x, y) \in \Omega_{L}$, then:

$$
\begin{aligned}
& \left(A(x, y)-g_{1}(x) S_{1}(x, y)-g_{2}(x, y) S_{2}(x, y)\right) \in \Sigma_{s}^{2 \times 2} \\
& S_{1}, S_{2} \in \Sigma_{s}^{2 \times 2} .
\end{aligned}
$$

Thus, conditions (52) and (53) are obtained if the following particular forms for $S_{1}$ and $S_{2}$ are considered:

$$
\begin{aligned}
& S_{1}=\left[\begin{array}{cc}
s_{1}(x, y) & 0 \\
0 & 0
\end{array}\right] \in \Sigma_{s}^{2 \times 2}, \quad s_{1} \in \Sigma_{s}, \\
& S_{2}=\left[\begin{array}{cc}
0 & 0 \\
0 & s_{2}(x, y)
\end{array}\right] \in \Sigma_{s}^{2 \times 2}, \quad s_{2} \in \Sigma_{s},
\end{aligned}
$$

the SOS matrix condition of which is immediately verified since $S_{1}=B B^{T}, S_{2}=C C^{T}$ with:

$$
\begin{aligned}
& B=\left[\begin{array}{cccc}
b_{1}(x, y) & \cdots & b_{m_{1}}(x, y) & 0 \\
0 & \cdots & 0 & 0
\end{array}\right] \in \mathbb{R}[\mathbf{x}]^{2 \times\left(m_{1}+1\right)}, \\
& C=\left[\begin{array}{cccc}
0 & 0 & \cdots & 0 \\
0 & c_{1}(x, y) & \cdots & c_{m_{2}}(x, y)
\end{array}\right] \in \mathbb{R}[\mathbf{x}]^{2 \times\left(m_{2}+1\right)},
\end{aligned}
$$

where $s_{1}=\sum_{j=1}^{m_{1}} b_{j}^{2}(x, y) \in \Sigma_{s}$ and $s_{2}=\sum_{j=1}^{m_{2}} c_{j}^{2}(x, y) \in$ $\Sigma_{s} ; b_{j} \in \mathbb{R}[\mathbf{x}], \forall j=1, \ldots, m_{1}, c_{j} \in \mathbb{R}[\mathbf{x}], \forall j=1, \ldots, m_{2}$, for some finite $m_{1} \in \mathbb{N}$ and $m_{2} \in \mathbb{N}$. Following the same arguments, condition (54) can be deduced from $A_{3}$ as in (59). On the other hand, (55) constrains the reactivity term $c=c(x)$ to satisfy the threshold of stability in (14), $\forall x \in \Omega$. Therefore, by means of the term $\int_{0}^{1} f(x) d x$, the optimization objective (41) minimizes the $\mathcal{L}^{2}$-norm of $K(1, y)$, for a selected precision $\Gamma_{0}$ in the approximate solution of the Kernel-PDE (34)-(35).

\section{Hyperbolic PIDE AND THE VOLTERRA-FredHOLM OPERATOR}

\section{A. Problem Setting}

In this section a class of first-order hyperbolic PIDEs (Partial Integral Differential Equations) with non-causal structure is considered (see details in [27], [28]); namely

$$
u_{t}(x, t)=u_{x}(x, t)+f(x) u(0, t)+\int_{0}^{x} h_{1}(x, y) u(y, t) d y
$$




$$
\begin{aligned}
& +\int_{x}^{1} h_{2}(x, y) u(y, t) d y, \\
u(1, t) & =U(t),
\end{aligned}
$$

where $u(x, 0)=u_{0}(x) \in \mathcal{C}(\Omega)$ is the initial condition and $f, h_{1}, h_{2}$ are real-valued continuous functions. The aim is to find a control action $U$ so that the origin of (66) is finite-time stable in the topology of the $\mathcal{L}^{2}$-norm. For this class of system, [27], [28] (see also [26] for parabolic systems) have proposed a Fredholm-type transformation (here referred to as Fredholm operator), namely

$$
\begin{aligned}
w(x, t) & =u(x, t)-\int_{0}^{x} P(x, y) u(y, t) d y-\int_{x}^{1} Q(x, y) u(y, t) d y, \\
& =\left(\mathbb{I}-\mathbb{F}_{P, Q}\right)[u(\cdot, t)](x),
\end{aligned}
$$

where $\mathbb{F}_{P, Q}: \mathcal{C}(\Omega ; \mathbb{R}) \rightarrow \mathcal{C}(\Omega ; \mathbb{R})$ is a linear operator in terms of Kernels $P$ and $Q$ in the lower $\Omega_{L}$ and upper $\Omega_{U}$ triangular domain, respectively, to transform the original system (66) into the target stable system:

$$
\begin{aligned}
w_{t}(x, t) & =w_{x}(x, t), \\
w(1, t) & =0,
\end{aligned}
$$

with a boundary feedback control determined by $U(t)=$ $\int_{0}^{1} P(1, y) u(y, t) d y$. Following the standard Backstepping PDE design procedure (detailed in [28]), the transformed system (66) takes the form:

$$
\begin{gathered}
w_{t}(x, t)-w_{x}(x, t)=\delta_{0}(x) u(0, t)-\delta_{3}(x) u(1, t) \\
+\int_{0}^{x} u(y, t) \delta_{1}(x, y) d y+\int_{x}^{1} u(y, t) \delta_{2}(x, y) d y,
\end{gathered}
$$

where the residual functions are:

$$
\begin{aligned}
\delta_{0}(x)= & f(x)+P(x, 0)-\int_{0}^{x} P(x, y) f(y) d y \\
& \quad-\int_{x}^{1} Q(x, y) f(y) d y, \quad \forall x \in[0,1], \\
\delta_{1}(x, y) & =h_{1}(x, y)+P_{x}(x, y)+P_{y}(x, y)-\int_{0}^{y} P(x, s) h_{2}(s, y) d s \\
- & \int_{y}^{x} P(x, s) h_{1}(s, y) d s-\int_{x}^{1} Q(x, s) h_{1}(s, y) d s, \forall(x, y) \in \Omega_{L},
\end{aligned}
$$

$\delta_{2}(x, y)=h_{2}(x, y)+Q_{x}(x, y)+Q_{y}(x, y)-\int_{0}^{x} P(x, s) h_{2}(s, y) d s$ - $\int_{x}^{y} Q(x, s) h_{2}(s, y) d s-\int_{y}^{1} Q(x, s) h_{1}(s, y) d s, \forall(x, y) \in \Omega_{U}$,

$\delta_{3}(x)=Q(x, 1)$.

Thus, the target system (68) is achievable if the continuous Kernels $P$ and $Q$ satisfy the so-called Kernel-PIDE ${ }^{4}$ :

$$
\begin{array}{rlrl}
\delta_{1}(x, y) & =0, & & \forall(x, y) \in \Omega_{L}, \\
\delta_{2}(x, y) & =0, & & \forall(x, y) \in \Omega_{U}, \\
\delta_{0}(x)=0, \delta_{3}(x) & =0, & \forall x \in \Omega .
\end{array}
$$

\footnotetext{
${ }^{4}$ For these coupled hyperbolic PIDEs, a method of analysis, computation and an equivalent sufficient (conservative) condition for a unique solution have been given in [28].
}

\section{B. Existence, Uniqueness and Invertibility}

In contrast to the Volterra Operator (13), existence, uniqueness and invertibility of the Fredholm operator (67) have been proved for specific conditions, most of them relying on the Banach contraction mapping principle (see [24] and references therein). In this context [27], [28] proposes a contraction mapping in terms of a system of integral equations equivalent to (74), which is used to calculate the Kernels by Picard's iterative method. This kind of (in some sense conservative) conditions can be circumvented, if the analysis is restricted to the space of continuous functions and Kernels with polynomials structure.

Lemma 2. If the solutions $u=u(\cdot, t)$ of the integral equation (67) are real-analytic functions, $\forall t \geq 0$, and the Kernels $P$ and $Q$ are polynomials (with bounded coefficients and finite degree), $P \neq Q$, then the homogeneous equation $\left(\mathbb{I}-\mathbb{F}_{P, Q}\right)[u(\cdot, t)](x)=0$ has only the trivial solution $u=0$.

Proof: Let $P(x, y)=\sum_{j=0}^{z\left(d_{P}\right)-1} p_{j} x^{\alpha_{j}} y^{\beta_{j}}$ and $Q(x, y)=$ $\sum_{j=0}^{z\left(d_{Q}\right)-1} q_{j} x^{\alpha_{j}} y^{\beta_{j}}$ be polynomials of degree $d_{P}, d_{Q} \in \mathbb{N}$, respectively, in accordance with (10); $d=\max \left\{d_{P}, d_{Q}\right\}, j=$ $0, \ldots, d, p_{j}=0, \forall\left(\alpha_{j}+\beta_{j}\right)>d_{P}$ or $q_{j}=0, \forall\left(\alpha_{j}+\beta_{j}\right)>d_{Q}$ as required. For each $t \geq 0$, since an analytic function has a unique series representation $u(x)=\sum_{k=0}^{\infty} a_{k} x^{k}$ [56] (without loss of generality, the analysis considers this function realanalytic at $x=0$ ), the homogeneous integral equation (67) (with $w=0$ ) can be formulated as:

$$
\begin{aligned}
\sum_{k=0}^{\infty} a_{k}\left(x^{k}+\sum_{j=0}^{s(d)-1} \frac{q_{j}-p_{j}}{\beta_{j}+k+1} x^{\alpha_{j}+\beta_{j}+k+1}\right. \\
\left.-\sum_{j=0}^{s(d)-1} \frac{q_{j}}{\beta_{j}+k+1} x^{\alpha_{j}}\right)=0 .
\end{aligned}
$$

This expression is equivalent to a linear (independent) combination of monomials of the polynomial basis $\Psi_{x}=$ $\left[1, x, \ldots, x^{d}, x^{d+1}, x^{d+2}, \ldots, x^{d+N+1}, \ldots\right]$; namely

$$
\sum_{k=0}^{\infty} x^{k}\left(\bar{\nu}_{k}\left[a_{0}, a_{1}, \ldots, a_{N}, \ldots\right]^{\top}\right)=0 \Leftrightarrow \Psi_{x} V \mathbf{a}=0,
$$

where $\mathbf{a}=\left[a_{0}, a_{1}, \ldots, a_{N}, \ldots\right]^{\top}$ and $\bar{\nu}_{k}=\left[\nu_{k, 0}, \ldots, \nu_{k, N}, \ldots\right]$ is the $k$-th row of the matrix:

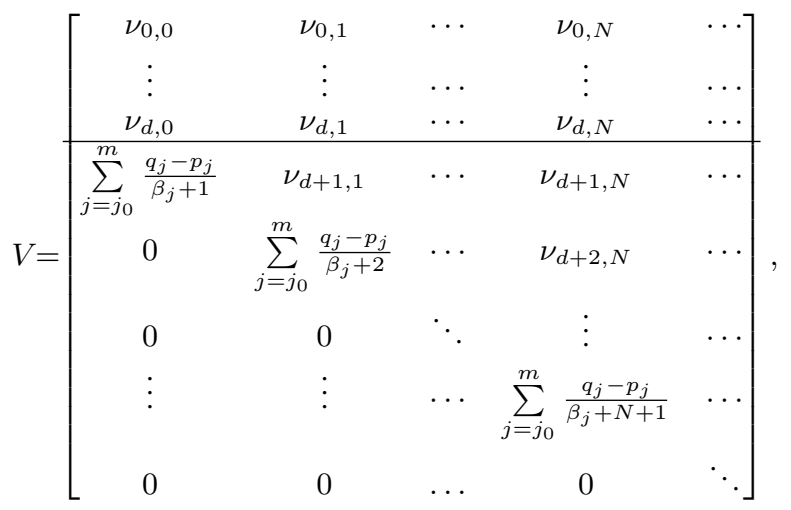

$\forall k \in \mathbb{N}, m=z(d)-1, j_{0}=m-d$, the elements of which are given by: 


$$
\begin{aligned}
\nu_{k, i}= & \chi_{[k]}(i)+\chi_{[0, d+k+1]}(i) \sum_{j=0}^{i-k-1} \frac{q_{s(i, j, k)}-p_{s(i, j, k)}}{j+k+1} \\
& -\chi_{[0, d]}(i) \sum_{j=i}^{d} \frac{q_{r(i, j)}}{j+k+1-i},
\end{aligned}
$$

with $\chi_{[a]}(i)=\{1, i=a ; 0, i \neq a\}$ and $\chi_{[a, b]}(i)=\{1, i \in$ $[a, b] ; 0, i \notin[a, b]\}$ indicator functions, $s(i, j, k)=j+(i-$ $k)(i-k-1) / 2, \forall i \geq k+1$ and $r(i, j)=j(j+3) / 2-i, \forall i \leq$ $d$ sub-indexes of polynomial coefficients. Thus, considering $u(x)=\sum_{k=0}^{N} a_{k} x^{k}$ as a finite series, due to the particular upper triangular structure of the matrix $V$ in (77), it is clear that $\operatorname{rank}(V)=N+1=\operatorname{dim}(\mathbf{a})$ (below the horizontal line of $V$, for $p_{j} \neq q_{j}, \forall j=j_{0}, \ldots, m$, only one diagonal element could be zero, i.e, there are at least $N$ independent rows. The extra row can be taken from above this line). In addition, it can be verified that $\nu_{d+k+1, d+k+1}=1, \forall k=0, \ldots, N$, so that $\lim _{N \rightarrow \infty} \operatorname{rank}(V) \rightarrow \operatorname{dim}(\mathbf{a})$. Therefore, the unique solution of (76) is the trivial one $\mathbf{a}=[0,0, \ldots]^{\top}$ [57], equivalent to $u(\cdot, t)=0, \forall t \geq 0$.

Lemma 3. If $f \in \mathcal{B}(\Omega \backslash\{0\}), \mathcal{B}=(\mathcal{C}-\mathcal{A})$, is a continuous real non-analytic function then $F(x)=x^{i} \int_{0}^{x} y^{j} f(y) d y$ is a continuous real non-analytic function, $\forall x \in(\Omega \backslash\{0\})$, with $i \in \mathbb{N}$ and $j \in \mathbb{N}$.

Proof: By contradiction (contrapositive), assuming $F(x)=x^{i} \int_{0}^{x} y^{j} f(y) d y \in \mathcal{A}(\Omega \backslash\{0\})$ as a continuous real analytic function, since $\frac{d F}{d x}(x) \in \mathcal{A}(\Omega \backslash\{0\})$ (derivative of a continuous real analytic function is continuous real analytic [56]), by the second fundamental theorem of calculus [58]:

$$
\begin{aligned}
& \mathcal{A}(\Omega \backslash\{0\}) \ni \frac{d F}{d x}(x)=i x^{i-1} \int_{0}^{x} y^{j} f(y) d y+x^{i+j} f(x), \\
& \mathcal{A}(\Omega \backslash\{0\}) \ni(\underbrace{x^{-(i+j)}}_{A_{1}} \underbrace{\frac{d F}{d x}(x)}_{A_{2}})= \\
& i \underbrace{\left(x^{-(i+j-1)}\right)}_{B_{1}} \underbrace{\left(x^{i} \int_{0}^{x} y^{j} f(y) d y\right)}_{B_{2}}+f(x) .
\end{aligned}
$$

Since the terms $A_{1}$ and $B_{1}$ are continuous real analytic functions in $\Omega \backslash\{0\}$ (functions with convergent Taylor's series and radius of convergence: $0<x<2 x_{0}, \forall x_{0} \in \Omega$ ), and by hypothesis $A_{2} \in \mathcal{A}(\Omega \backslash\{0\})$ and $B_{2} \in \mathcal{A}(\Omega \backslash\{0\})$, based on the property that the product of analytic functions is also an analytic function [56], it is inferred that $f \in \mathcal{A}(\Omega \backslash\{0\})$.

Lemma 4. If the solutions of the integral equation (67) $u=$ $u(\cdot, t) \in \mathcal{C}(\Omega), \forall t \geq 0$, and the Kernels $P$ and $Q$ are polynomials (with bounded coefficients and finite degree), $P \neq$ $Q$, then the homogeneous equation $\left(\mathbb{I}-\mathbb{F}_{P, Q}\right)[u(\cdot, t)](x)=0$ has only the trivial solution $u=0$.

Proof: Consider $u=u(\cdot, t) \in \mathcal{B}(\Omega), \forall t \geq 0$ with $\mathcal{B}=(\mathcal{C}-\mathcal{A})$ (the case $u \in \mathcal{A}(\Omega)$ has been proved in Lemma 2), with $\mathbb{F}_{P, Q}: \mathcal{C}(\Omega ; \mathbb{R}) \rightarrow \mathcal{C}(\Omega ; \mathbb{R})$ as in (67). For polynomial Kernels $P \neq Q$ formulated according the standard bivariate basis (10), with polynomial degrees $\operatorname{deg}(P)=d_{P}$, $\operatorname{deg}(Q)=d_{Q}, d=\max \left(d_{P}, d_{Q}\right)$, it is straightforward to verify that the homogeneous equation $\left(\mathbb{I}-\mathbb{F}_{P, Q}\right)[u(\cdot, t)](x)=0$ can be written as ${ }^{5}$ :

$$
u(x)-\underbrace{\sum_{n=0}^{d} \psi_{n}(x) \int_{0}^{x} y^{n} u(y) d y}_{T_{1}}-\underbrace{\sum_{i=0}^{d_{Q}} \theta_{i} x^{i}}_{T_{2}}=0,
$$

with

$$
\begin{aligned}
\theta_{i} & =\sum_{k=i}^{d_{Q}} q_{s(k, i)}\left(\int_{0}^{1} y^{k-i} u(y) d y\right) \\
\psi_{n}(x) & =\sum_{k=n}^{d} \underbrace{\left(q_{r(k, n)}-p_{r(k, n)}\right)}_{c_{r(k, n)}} x^{k-n}, \\
s(k, i) & =k(k+3) / 2-i, \quad r(k, n)=n+k(k+1) / 2 .
\end{aligned}
$$

As for the term $T_{1}$, consider the following set of functions:

$$
\begin{aligned}
\Theta= & \left\{\psi_{0}(x), \psi_{1}(x) y, \ldots, \psi_{n}(x) y^{n}, \ldots, \psi_{d-1}(x) y^{d-1}, \psi_{d}(x) y^{d}\right\} \\
= & \left\{\left(c_{0}+c_{1} x+c_{3} x^{2}+\ldots+c_{r(d, 0)} x^{d}\right)\right. \\
& \left(c_{2}+c_{4} x+c_{7} x^{2}+\ldots+c_{r(d, 1)} x^{d-1}\right) y, \ldots \\
& \left(\sum_{k=n}^{d} c_{r(k, n)} x^{k-n}\right) y^{n}, \ldots \\
& \left.\left(c_{r(d-1, d-1)}+c_{r(d, d-1)} x\right) y^{d-1}, c_{r(d, d)} y^{d}\right\}
\end{aligned}
$$

Since $\Theta$ is a linearly independent (LI) set of functions (bivariate polynomials of different degrees),

$\bar{\Theta}=\Theta \circ u(y)=\left\{\psi_{0}(x), \ldots, \psi_{n}(x) y^{n}, \ldots, \psi_{d}(x) y^{d}\right\} \circ u(y)$,

is also a LI set of functions $\forall u \in \mathcal{C}(\Omega), u(x, t) \neq 0, \forall x \in \Omega$, $t \geq 0$, where $\circ$ denotes the Hadamard product (element-wise).

Let $\mathbb{V}: \mathcal{C}(\Omega ; \mathbb{R}) \rightarrow \mathcal{C}(\Omega ; \mathbb{R})$ be the standard Volterra operator given by: $\mathbb{V}[f(\cdot, t)](x)=\int_{0}^{x} f(y, t) d y, f \in \mathcal{C}(\Omega)$. Due to the Volterra operator $\mathbb{V}$ is linear and injective [59], this maps (83) to

$$
\begin{aligned}
& \Gamma_{1}=\mathbb{V}(\bar{\Theta})=\left\{\left(c_{0}+c_{1} x+\ldots+c_{r(d, 0)} x^{d}\right) \int_{0}^{x} u(y) d y, \ldots,\right. \\
& \left.\left(\sum_{k=n}^{d} c_{r(k, n)} x^{k-n}\right) \int_{0}^{x} y^{n} u(y) d y, \ldots, c_{r(d, d)} \int_{0}^{x} y^{d} u(y) d y\right\}
\end{aligned}
$$

which also is a set of LI functions (a linear injective operator preserves linear independence) [60]. In addition, based on Lemma 3, restricting the domain $\Omega$ to $\bar{\Omega}=\Omega \backslash\{0\}, \Gamma_{1}$ is a set of continuous real non-analytic functions.

Thus, since the term $T_{2}$ in (81) is a linear combination of elements of the standard LI univariate polynomial basis $\Gamma_{2}=$

\footnotetext{
${ }^{5}$ For notational simplicity the time-dependence in the functions is dropped.
} 
$\left\{1, x, x^{2}, \ldots, x^{d_{Q}}\right\}$, and this basis cannot span the space $\mathcal{B}(\bar{\Omega})$, it is inferred that $\Gamma_{1} \cup \Gamma_{2}$ is a set of LI functions, $\forall x \in \bar{\Omega}$.

Moreover, it is immediate to verify that if $u \in \mathcal{B}(\bar{\Omega}), u$ is not a linear combination of the elements of $\Gamma_{2}$. Similarly, regarding $\Gamma_{1}$, for $\alpha_{1}, \alpha_{2} \in \mathbb{R}, \alpha_{1} u(x)+\alpha_{2} x^{j} \int_{0}^{x} y^{n} u(y)=0 \Leftrightarrow \alpha_{1}=$ $\alpha_{2}=0$ or $u=0, \forall x \in \bar{\Omega}$, since it is an homogeneous Volterra integral equation of the second kind (this can also be obtained including $u_{y}$ to $\Theta$ and then applying $\mathbb{V}$. However it requires $u \in \mathcal{C}^{1}$ ). Therefore, $\Gamma=\{u(x)\} \cup \Gamma_{1} \cup \Gamma_{2}$ is a set of LI functions in $\bar{\Omega}$.

Finally, since $u=0$ is solution of (80) for $x=0\left(\theta_{0}=0\right)$, and (80) is a linear combination of LI functions in $\Gamma$, with $u \in \mathcal{B}(\Omega), \forall x \in \bar{\Omega}$, and considering Lemma 2 , it is concluded that $(\mathbb{I}-\mathbb{F})[u(\cdot, t)](x)=0$ has only the trivial solution $u=$ $u(\cdot, t)=0$, with $u \in \mathcal{C}(\Omega), \forall x \in \Omega, \forall t \geq 0$.

Theorem 2. Let $\mathbb{T}=\mathbb{I}-\mathbb{F}_{P, Q}: \mathcal{X} \rightarrow \mathcal{X}$ be the linear operator in (67), where $\mathcal{X}=\mathcal{X}_{\mathcal{C}}=\left(\mathcal{C}(\Omega ; \mathbb{R}),\|\cdot\|_{\infty}\right)$ is the Banach space of continuous functions or $\mathcal{X}=\mathcal{X}_{\mathcal{H}}=(\mathcal{A} \subset$ $\left.\mathcal{L}_{2}(\Omega ; \mathbb{R}),\langle\cdot, \cdot\rangle_{\mathcal{L}^{2}}\right)$ is the Hilbert space of square integrable real-analytic functions. If $u=u(\cdot, t) \in \mathcal{X}, \forall t \geq 0$, and the Kernels $P$ and $Q$ are polynomials (with bounded coefficients and finite degree), $P \neq Q$, then the integral equation $\mathbb{T}[u(\cdot, t)](x)=w(x, t)$ (67) has a unique solution and the operator $\mathbb{T}$ is boundedly invertible in $\mathcal{X}$.

Proof: Let $\mathbb{F}_{P, Q}$ be the linear operator with $P$ and $Q$ bivariate polynomials (bounded coefficients) as in (10). Reordering terms, these polynomials can be alternatively expressed:

$$
\begin{aligned}
& P(x, y)=\sum_{n=0}^{d_{P}} x^{n} \varphi_{n}(y), \varphi_{n}(y)=\sum_{k=n}^{d_{P}} p_{s(k, n)} y^{k-n} \\
& Q(x, y)=\sum_{n=0}^{d_{Q}} x^{n} \psi_{n}(y), \psi_{n}(y)=\sum_{k=n}^{d_{Q}} q_{s(k, n)} y^{k-n}
\end{aligned}
$$

where $s(k, n)=k(k+3) / 2-n$. Taking $d=\max \left\{d_{P}, d_{Q}\right\}$ and assigning $p_{s(k, n)}=0, \forall s(k, n)>z\left(d_{P}\right)-1$ or $q_{s(k, n)}=$ $0, \forall s(k, n)>z\left(d_{Q}\right)-1$ as required, the linear operator can be written as:

$$
\begin{array}{r}
\mathbb{F}_{P, Q}[u(\cdot, t)](x)=\int_{0}^{1} \sum_{n=0}^{d} \sum_{k=n}^{d}\left(\chi_{[0, x]}(y) p_{s(k, n)}+\right. \\
\left.\chi_{[x, 1]}(y) q_{s(k, n)}\right) x^{n} y^{k-n} u(y, t) d y \\
=\int_{0}^{1} F(x, y) u(y, t) d y=\sum_{n=0}^{d} x^{n} \int_{0}^{1} \bar{F}(n, y) u(y, t) d y
\end{array}
$$

where $\chi_{[a, b]}(y)=\{1, y \in[a, b] ; 0, y \notin[a, b]\}$ is the indicator function $([a, b] \subset \mathbb{R})$ and $\bar{F}=\sum_{k=n}^{d} y^{k-n}\left(\chi_{[0, x]}(y) p_{s(k, n)}+\right.$ $\left.\chi_{[x, 1]}(y) q_{s(k, n)}\right)$. Since $F$ is a bounded polynomial Kernel (a special class of degenerate Kernels [23]), it is immediate that $\mathbb{F}_{P, Q}$ is a finite rank operator, as (87) shows ${ }^{6}$. Its compactness can be proved in some Banach and Hilbert spaces. In the case of the Banach space of continuous functions, since $F$ is bounded in $S=[0,1]^{2}$ and continuous except possibly along the curve $x=y$ (also known as mildly discontinuous Kernel [62]), $\mathbb{F}_{P, Q}$ is a compact operator [39], [61] (also denominated

\footnotetext{
${ }^{6} \mathbb{F}_{P, Q}$ maps into a finite-dimensional space [23], [37], [61].
}

as completely continuous operator [41]). In the Hilbert space of square integrable functions, since $\mathbb{F}_{P, Q}$ is a finite rank operator [16], it is a Hilbert-Schmidt operator $\left(\mathbb{F}_{P, Q}[u](\cdot, t)=\right.$ $\sum_{j=0}^{d-1} \psi_{j}(y)\left\langle\phi_{j}(x), u(y, t)\right\rangle_{\mathcal{L}^{2}(\Omega)}$, for some $\left\{\phi_{j}\right\},\left\{\psi_{j}\right\}$ finite orthonormal systems in $\mathcal{L}^{2}(\Omega)$ ) and therefore compact [38], [41], [61], [63]. Thus, based on Lemma 2, Lemma 4 and on the property of compactness of $\mathbb{F}_{P, Q}$, according to a particular feature of the Fredholm Alternative Theorem ([23, Corollary $3.5],[37 \text {, Corollary 7.27] })^{7}$, the solution of (67) is unique and the operator $\mathbb{T}$ is boundedly invertible in $\mathcal{X}_{\mathcal{C}}$ and $\mathcal{X}_{\mathcal{H}}$.

\section{Kernel-PIDE as a Convex Optimization Problem}

\section{Proposition 5. Let}

$u(x, t)=w(x, t)+\int_{0}^{x} R(x, y) w(y, t) d y+\int_{x}^{1} S(x, y) w(y, t) d y$

be the inverse transformation of (67) in terms of the Kernels $R$ and $S$ (as it is proposed in [26], [27], [28] under specific conditions on the system (66)). Let $\Delta_{1}=\int_{0}^{1} \int_{0}^{x} \delta_{1}^{2}(x, y) d y d x$ and $\Delta_{2}=\int_{0}^{1} \int_{x}^{1} \delta_{2}^{2}(x, y) d y d x$ be the mean square of the residual functions (71) and (72), respectively. Considering $\delta_{0}(x)=0$ and $\delta_{3}(x)=0$ in (70)-(73), the transformed system (69) is exponentially stable in $\mathcal{L}^{2}$-norm topology if the residual functions satisfy:

$$
\sqrt{\Delta_{1}}+\sqrt{\Delta_{2}} \leq \frac{e^{-1}}{\left(1+\sqrt{\sigma_{1}}+\sqrt{\sigma_{2}}\right)},
$$

where $\sigma_{1}=\int_{0}^{1} \int_{0}^{x} R^{2}(x, y) d y d x$ and $\sigma_{2}=\int_{0}^{1} \int_{x}^{1} S^{2}(x, y) d y d x$.

Proof: See the Appendix.

Based on this result, similarly to Proposition 2, a relaxation on the zero matching condition for the residual functions $\delta_{1}$ and $\delta_{2}$ can be considered and the Kernel-PIDE (74) can be solved approximately in terms of polynomial Kernels.

Proposition 6. Let $N(x, y)=\sum_{k=0}^{z(d)-1} n_{k} x^{\alpha_{k}} y^{\beta_{k}}$ and $M(x, y)=\sum_{k=0}^{z(d)-1} m_{k} x^{\alpha_{k}} y^{\beta_{k}}$ be polynomial approximations of $P$ and $Q$, respectively, of arbitrary even degree $d \in \mathbb{N}$, with coefficients $n_{k}$ and $m_{k}$ and powers in accordance with (10). Let $\delta_{1}=\delta_{1}(x, y)$ and $\delta_{2}=\delta_{2}(x, y)$ be the resulting residual functions according to (71) and (72), respectively, with degree $d_{\delta}=2\left\lceil\left(\max \left\{d+d_{h_{1}}, d+d_{h_{2}}\right\}+1\right) / 2\right\rceil$ and $\gamma_{1} \geq 0, \gamma_{2} \geq 0$. For any functions $f, h_{1}, h_{2}$ described by polynomials of degree $d_{f}, d_{h_{1}}, d_{h_{2}}$, respectively, the KernelPIDE (74) can be formulated as the convex optimization problem $^{8}$ :

$$
\underset{\gamma_{j}, N, M, T_{1}, T_{2}, s_{j}}{\operatorname{minimize:}} \quad \gamma_{1}+\gamma_{2}
$$

subject to:

$$
\left[\begin{array}{cc}
2 \gamma_{1}-T_{1}(x, y)-s_{1}(x, y) g_{1}(x) & \delta_{1}(x, y) \\
\delta_{1}(x, y) & \gamma_{1}-s_{2}(x, y) g_{2}(x, y)
\end{array}\right] \in \Sigma_{s}^{2 \times 2}
$$

\footnotetext{
${ }^{7}$ The uniqueness of $\mathbb{F}_{P, Q} u=u$ (trivial solution) implies the existence of the solution of $\left(\mathbb{I}-\mathbb{F}_{P, Q}\right)[u(\cdot, t)](x)=w(x, t)$.

${ }^{8}$ The expression $\delta=\delta(\mathbf{x}) \mid \underset{Q \approx N}{P \approx N}$ indicates that in the function $\delta$, the Kernels $P$ and $Q$ has been substituted by the polynomials $N$ and $M$, respectively.
} 


$$
\begin{aligned}
& {\left[\begin{array}{cc}
2 \gamma_{2}-T_{2}(x, y)-s_{3}(x, y) g_{1}(x) & \delta_{2}(x, y) \\
\delta_{2}(x, y) & \gamma_{2}-s_{4}(x, y) g_{3}(x, y)
\end{array}\right] \in \Sigma_{s}^{2 \times 2},} \\
& s_{1}, s_{2}, s_{3}, s_{4} \in \Sigma_{s}, \\
& \int_{0}^{1} \int_{0}^{x} T_{1}(x, y) d y d x=0, \int_{0}^{1} \int_{x}^{1} T_{2}(x, y) d y d x=0 \\
& \delta_{1}=\left.\delta_{1}(x, y)\right|_{Q \approx N} ^{P \approx N} \quad \text { as in (71), } \\
& \delta_{2}=\left.\delta_{2}(x, y)\right|_{Q \approx M} ^{P \approx N} \quad \text { as in (72), } \\
& \delta_{0}=\left.\delta_{0}(x)\right|_{Q \approx N} ^{P \approx N}=0 \quad \text { as in (70), } \\
& \delta_{3}=\left.\delta_{3}(x)\right|_{Q \approx M}=M(x, 1)=0 \text { as in (73), }
\end{aligned}
$$

for some polynomials $s_{j}, j=1, \ldots, 4$ of degree $d_{\delta}-2, T_{1}$ and $T_{2}$ of degree $2 d_{\delta}, g_{1}(x)=x(1-x), g_{2}(x, y)=y(x-y)$ and $g_{3}(x, y)=(1-y)(y-x)$. The optimal root mean square bounds of the residual functions are: $\sqrt{\Delta_{1}} \leq \gamma_{1}$ and $\sqrt{\Delta_{2}} \leq \gamma_{2}$.

Proof: The convex optimization problem formulation follows similar arguments as the ones given in the proof of Proposition 2. Regarding the optimal mean square bounds for $\delta_{1}$ and $\delta_{2}$, let

$$
A_{1}=\left[\begin{array}{cc}
2 \gamma_{1}-T_{1}(x, y) & \delta_{1}(x, y) \\
\delta_{1}(x, y) & \gamma_{1}
\end{array}\right] \succ 0, \forall(x, y) \in \Omega_{L}
$$

be a symmetric real polynomial positive definite matrix on $\Omega_{L}$ (pointwise condition). Taking the Schur's complement of $A_{1}$, its integration on the domain $\Omega_{L}$ yields

$$
\begin{aligned}
& A_{1} \succ 0 \Leftrightarrow 2 \gamma_{1}^{2}-\gamma_{1} T_{1}(x, y)-\delta_{1}^{2}(x, y)>0, \forall(x, y) \in \Omega_{L}, \\
& \int_{0}^{1} \int_{0}^{x} \delta_{1}^{2}(x, y) d y d x<\gamma_{1}^{2}-\gamma_{1} \int_{0}^{1} \int_{0}^{x} T_{1}(x, y) d y d x .
\end{aligned}
$$

If there exists a polynomial function $T_{1}$ satisfying (94), it is clear that $\gamma_{1}$ is an upper bound of the root mean square value of $\delta_{1}$ in $\Omega_{L}$. The matrix conditions (91)-(92) are obtained via the matrix-polynomial version of Putinar's Positivstellensatz as it has been detailed in the proof of Proposition 4. Following the same arguments, conditions for $\delta_{2}$ in $\Omega_{U}$ can be deduced. Therefore, according to the optimization objective (90), the root mean square error of $\delta_{1}$ and $\delta_{2}$ are minimized.

\section{Approximate Inverse Transformation}

For known Kernels $P$ and $Q$, the inverse transformation of (67) can be found by means of the direct substitution of (88) in (67), which yields

$$
\int_{0}^{x} w(y, t) \delta_{1}(x, y) d y+\int_{x}^{1} w(y, t) \delta_{2}(x, y) d y=0,
$$

with the equality satisfied if the residual functions

$$
\begin{aligned}
& \delta_{1}(x, y)=R(x, y)-P(x, y)-\int_{0}^{y} P(x, s) S(s, y) d s \\
& -\int_{y}^{x} P(x, s) R(s, y) d s-\int_{x}^{1} Q(x, s) R(s, y) d y, \forall(x, y) \in \Omega_{L}, \\
& \delta_{2}(x, y)=S(x, y)-Q(x, y)-\int_{0}^{x} P(x, s) S(s, y) d s \\
& -\int_{x}^{y} Q(x, s) S(s, y) d s-\int_{y}^{1} Q(x, s) R(s, y) d s, \forall(x, y) \in \Omega_{U},
\end{aligned}
$$

are identically zero in their respective triangular domains. Since (101) does not depend on any original and target systems, it can be used to find an approximation of the inverse Kernels $R$ and $S$, given the approximate direct ones $P \approx N$, $Q \approx M$.

Proposition 7. Let $A(x, y)=\sum_{k=0}^{z(d)-1} a_{k} x^{\alpha_{k}} y^{\beta_{k}}$ and $B(x, y)=\sum_{k=0}^{z(d)-1} b_{k} x^{\alpha_{k}} y^{\beta_{k}}$ be the polynomial approximations of $R$ and $S$, respectively, of arbitrary even degree $d \in \mathbb{N}$, with coefficients $a_{k}$ and $b_{k}$ and powers in accordance with (10). Let $\delta_{1}=\delta_{1}(x, y)$ and $\delta_{2}=\delta_{2}(x, y)$ be the resulting residual functions according to (102) and (103), respectively, with degree $d_{\delta}=2\left\lceil\left(\max \left\{d_{N}, d_{M}\right\}+d+1\right) / 2\right\rceil$ and $\gamma_{j} \geq$ $0, j=1, \ldots, 4$. For given direct approximate Kernels $N$ and $M$ of $P$ and $Q$ with degrees $d_{N}$ and $d_{M}$, respectively (solution of (90)-(98)), the integral equation (101)-(103) can be formulated as the convex optimization problem:

$$
\begin{aligned}
& \underset{\gamma_{j}, B, s_{j}}{\operatorname{minimize}} \quad \gamma_{1}+\gamma_{2}+\gamma_{3}+\gamma_{4} \\
& \text { subject to: } \\
& \left(\delta_{1}(x, y)-\underline{\rho}_{1}-\left[s_{1}(x, y) s_{2}(x, y)\right] g_{L}(x, y)\right) \in \Sigma_{s}, \\
& \left(\bar{\rho}_{1}-\delta_{1}(x, y)-\left[s_{3}(x, y) s_{4}(x, y)\right] g_{L}(x, y)\right) \in \Sigma_{s}, \\
& \left(\delta_{2}(x, y)-\underline{\rho}_{2}-\left[s_{5}(x, y) s_{6}(x, y)\right] g_{U}(x, y)\right) \in \Sigma_{s} \text {, } \\
& \left(\bar{\rho}_{2}-\delta_{2}(x, y)-\left[s_{7}(x, y) s_{8}(x, y)\right] g_{U}(x, y)\right) \in \Sigma_{s} \text {, } \\
& s_{1}, s_{2}, s_{3}, s_{4}, s_{5}, s_{6}, s_{7}, s_{8} \in \Sigma_{s} \text {, } \\
& {\left[\begin{array}{ll}
\gamma_{1} & \underline{\rho}_{1} \\
\underline{\rho}_{1} & \gamma_{1}
\end{array}\right] \succeq 0, \quad\left[\begin{array}{ll}
\gamma_{2} & \bar{\rho}_{1} \\
\bar{\rho}_{1} & \gamma_{2}
\end{array}\right] \succeq 0,} \\
& {\left[\begin{array}{ll}
\gamma_{3} & \underline{\rho}_{2} \\
\underline{\rho}_{2} & \gamma_{3}
\end{array}\right] \succeq 0, \quad\left[\begin{array}{ll}
\gamma_{4} & \bar{\rho}_{2} \\
\bar{\rho}_{2} & \gamma_{4}
\end{array}\right] \succeq 0,}
\end{aligned}
$$

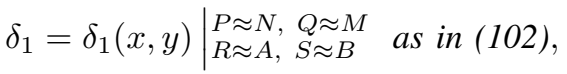

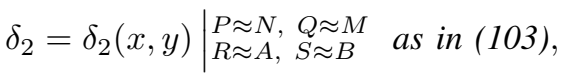

for some polynomials $s_{j}, j=1, \ldots, 8$ of degree $d_{\delta}-2, g_{L}=$ $\left[g_{1}, g_{2}\right], g_{U}=\left[g_{1}, g_{3}\right], g_{1}(x)=x(1-x), g_{2}(x, y)=y(x-y)$ and $g_{3}(x, y)=(1-y)(y-x)$. The optimal minimal bounds for the residual functions are: $\overline{\delta_{1}}=\max \left\{\gamma_{1}, \gamma_{2}\right\}$ and $\overline{\delta_{2}}=$ $\max \left\{\gamma_{3}, \gamma_{4}\right\}$.

Proof: The proof follows the same arguments of the one of Proposition 2, hence it is omitted.

\section{NUMERICAL RESUlts}

The numerical solution of the convex optimization problems proposed in this article has been obtained via the Yalmip toolbox for Matlab [64] using the SDP package part of the Mosek solver [65].

\section{A. Parabolic PDE with constant reactivity term}

To illustrate the precision of the method proposed with respect to the polynomial degree selected for the Kernels, this example considers the case of $\lambda=20$ and $\epsilon=1$ in the system (12) and $c=0$ in the target system (14). The Kernel $K$ in (13) is approximated solving the convex optimization problem (22)(32). The bounds of the residual functions $\delta_{1}$ and $\delta_{2}$, and the approximation error with respect to the closed-form solution (17) are depicted in Figure 1. 


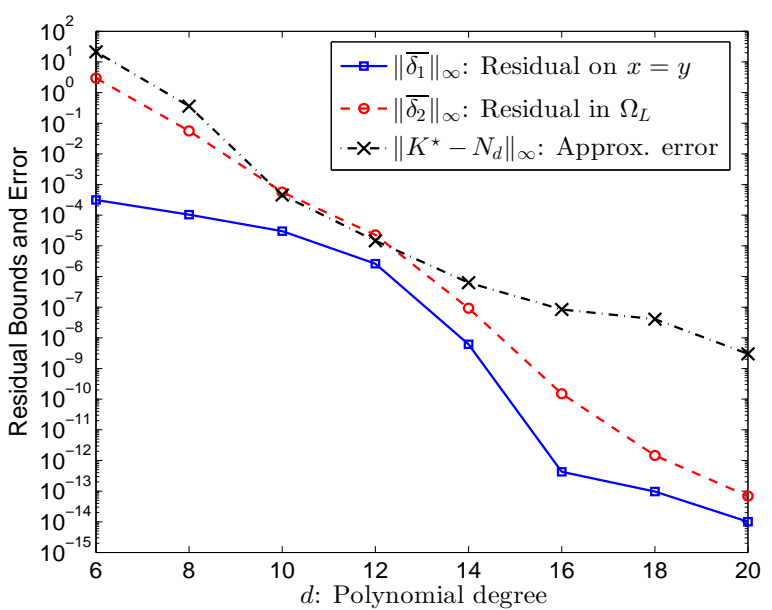

Fig. 1: Bounds for the residual functions in (15), solution of (22)-(32), as a function of polynomial degrees $d$.

\section{B. Parabolic PDE: Target System Optimization}

This example considers the optimization problem described in Section IV for the system (12) with $\epsilon=1$ and two cases for the reactivity term $\lambda . \underline{\mathrm{C} 1}: \lambda=\lambda_{1}(x)=25-80(x-0.5)^{2}, \underline{\mathrm{C} 2}$ : $\lambda=\lambda_{2}(x)=80(x-0.3)(x-0.7)-2$, which are plotted in Figure 2 (a). The numerical optimization has been carried out via Proposition 4 using a polynomial Kernel $N=N(x, y)$ of degree $d=16$ to approximate $K$ in (13), a polynomial function for the reactivity coefficient $c=c(x)$ in (14) of degree $d_{c}=10$ and weight factors $\sigma_{0}=10, \sigma_{1}=1$ and $\sigma_{2}=15$ in the cost function (41); $\Gamma_{0}=10^{-6}$ as precision in (47) and $\Gamma_{1}=-0.5\left(\pi^{2} / 4\right)$ to guarantee the threshold of stability for $c$ in (14). Figure 2(a) shows the optimal polynomials functions $c_{1}^{\text {opt }}$ and $c_{2}^{\text {opt }}$ determined for the cases $\mathrm{C} 1$ and $\mathrm{C} 2$, respectively. Figure 2(b) depicts the polynomial Kernels $N(1, y)$ obtained for both cases, considering $c=0$ and the optimal functions found for the target system (14).

\section{Hyperbolic PIDE: Fredholm-type Operator}

This example considers the problem presented in [27][28]:

$$
\begin{aligned}
f(x) & =a+\frac{b \sigma}{\sqrt{c} \cosh (\sqrt{c})} \sinh (\sqrt{c}(1-x)) \\
h_{2}(x) & =-\frac{b \sigma}{\cosh (\sqrt{c})} \cosh (\sqrt{c} x) \cosh (\sqrt{c}(1-y)) \\
h_{1}(x) & =h_{2}(x)+b \sigma \cosh (\sqrt{c}(x-y))
\end{aligned}
$$

with $a=1.25, b=0.1, c=0.1, \sigma=10$ (the application of the proposed approach is not limited to this case, which has been selected for comparison purposes). This problem (equations (71)-(73) of [28]) corresponds to a first-order PDE coupled with a second order ODE, equivalent to the 1dimensional hyperbolic PDE (66). The direct Kernels $P$ and $Q$ in (67) have been approximated solving the convex optimization problem (90)-(98). To implement this approach, the functions $f, h_{1}$ and $h_{2}$ in (114)-(116) have been approximated by a combination of univariate polynomials of degree 4 , with maximum approximation error $<4.2 \cdot 10^{-8}$. The approximate Kernels $M$ and $N$ for a polynomial degree $d=10$ are shown in Figure 3(a), with root mean square bounds for the residual functions: $\gamma_{1}=4.70 \cdot 10^{-10}$ and $\gamma_{2}=1.07 \cdot 10^{-9}$. Using
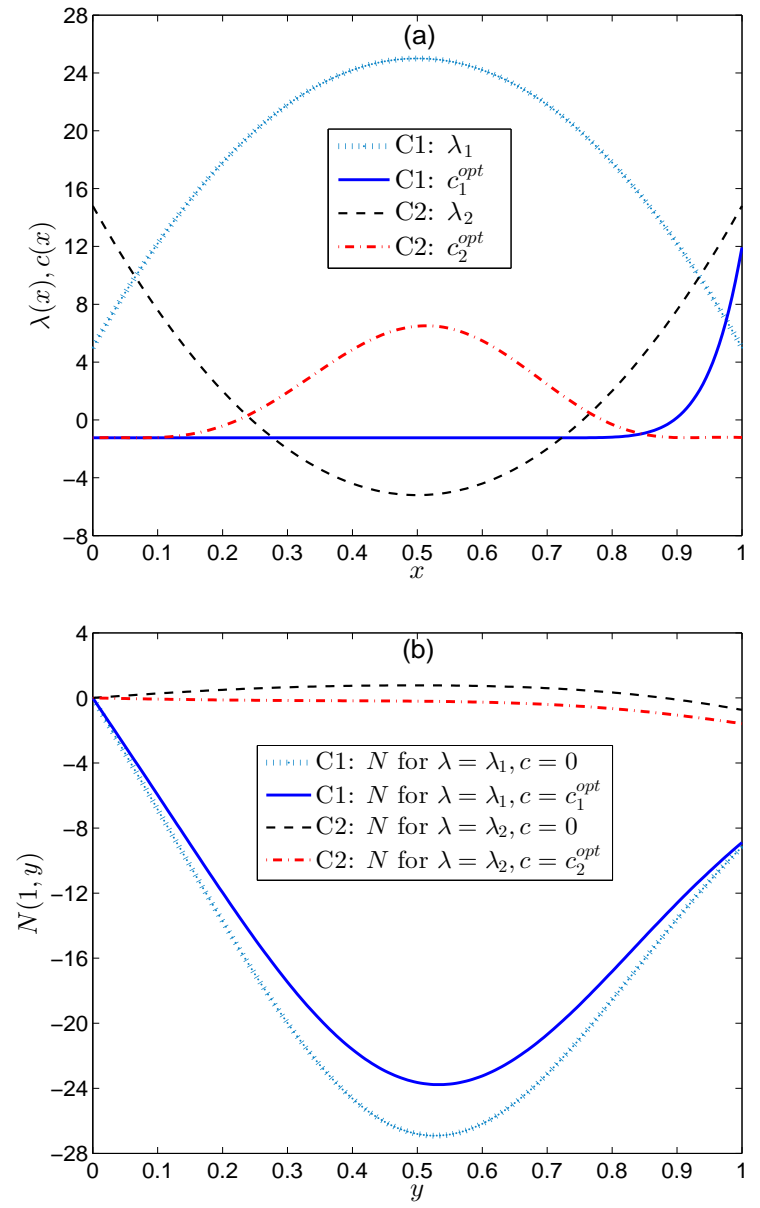

Fig. 2: Target System Optimization for cases C1 and C2. (a) Reactivity coefficients in (12) and optimal polynomials functions $c=c(x)$ in (14) determined via Proposition 4. (b) Resulting polynomial Kernels $N(1, y)$.

the previous result, the inverse Kernels $R$ and $S$ have been approximated solving (104)-(113) for a polynomial degree $d=10$. The result is shown in Figure 3(b) with bounds of the residual functions: $\bar{\gamma}=\max \left\{\gamma_{1}, \gamma_{2}, \gamma_{3}, \gamma_{4}\right\} \leq 8.01 \cdot 10^{-10}$.

\section{Discontinuous Kernels: Linear Coupled Hyperbolic PDEs}

This example considers the set of Kernel-PDEs derived from the motion planning problem for hyperbolic PDEs presented in Section V.C of [66], namely

$$
\begin{aligned}
& \mu_{1} \frac{\partial L_{11}(x, y)}{\partial x}+\mu_{1} \frac{\partial L_{11}(x, y)}{\partial y}=\sigma_{21} L_{12}(x, y) \\
& \mu_{1} \frac{\partial L_{12}(x, y)}{\partial x}+\mu_{2} \frac{\partial L_{11}(x, y)}{\partial y}=\sigma_{12} L_{12}(x, y) \\
& L_{11}(x, 0)=L_{12}(x, 0)=0, L_{12}(x, x)=\frac{\sigma_{12}}{\mu_{2}-\mu_{1}} \\
& \mu_{2} \frac{\partial L_{21}(x, y)}{\partial x}+\mu_{1} \frac{\partial L_{21}(x, y)}{\partial y}=\sigma_{21} L_{22}(x, y) \\
& \mu_{2} \frac{\partial L_{22}(x, y)}{\partial x}+\mu_{2} \frac{\partial L_{22}(x, y)}{\partial y}=\sigma_{12} L_{21}(x, y) \\
& L_{22}(x, 0)=0, L_{21}(x, x)=\frac{\sigma_{21}}{\mu_{1}-\mu_{2}}, L_{21}(1, y)=l(y)
\end{aligned}
$$



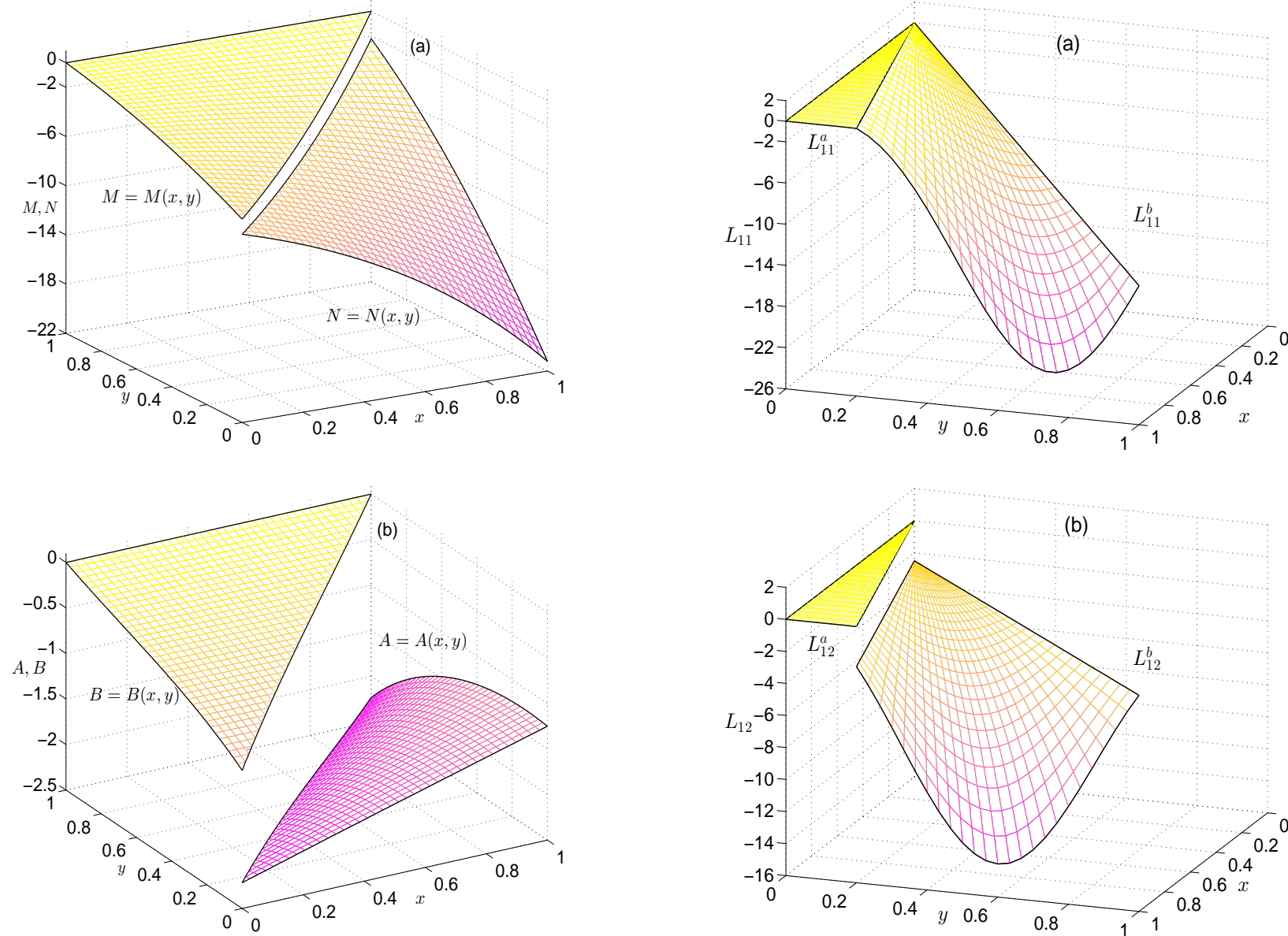

Fig. 3: (a) Direct approximate Kernels $M$ and $N$ for $d=10$ $(P \approx N, Q \approx M$ in (67)). (b) Inverse approximate Kernels $A$ and $B$ for $d=10(R \approx A, S \approx B$ in (88)).

$\forall(x, y) \in \Omega_{L}$, with Kernels $L_{i j} \in \mathcal{L}^{\infty}\left(\Omega_{L}\right), i=1,2, j=$ 1,2 , for an arbitrary function $l=l(y)$ satisfying $l(1)=$ $\sigma_{21} /\left(\mu_{1}-\mu_{2}\right) ; \mu_{1}=1, \mu_{2}=0.2, \sigma_{12}=2$ and $\sigma_{21}=5$. As it has been shown in [66], these Kernel-PDEs can have a closed-form solution in terms of Bessel functions (equations (101)-(104) in [66]).

Regarding the problem (117)-(119), the resulting solution $L_{12}$ is discontinuous along the line $y=\left(\mu_{2} / \mu_{1}\right) x$ whereas $L_{11}$ is continuous, both with zero value $\forall x \in[0,1], y \in$ $\left[0,\left(\mu_{2} / \mu_{1}\right) x\right]$. Due to this piecewise continuous characteristic, the method proposed based on polynomial Kernels cannot be applied directly. To obtain a solution the information about the lines of discontinuity has to be consider to define sub-domains of $\Omega_{L}$ where separate Kernel-PDEs can be posed, including suitable boundary conditions. For instance, for $\Omega_{L}=\Omega_{a} \cup \Omega_{b}$, Proposition 2 can be used in each sub-domain, namely Kernels $L_{11}^{a}$ and $L_{12}^{a}$ in $\Omega_{a}=\left\{(x, y) \in \mathbb{R}^{2} ; x \in[0,1], y \in\right.$ $\left.\left[0,\left(\mu_{2} / \mu_{1}\right) x\right]\right\}$ with $L_{11}^{a}(x, 0)=L_{12}^{a}(x, 0)=0 ; L_{11}^{b}$ and $L_{12}^{b}$ in $\Omega_{b}=\left\{(x, y) \in \mathbb{R}^{2} ; x \in[0,1], y \in\left[\left(\mu_{2} / \mu_{1}\right) x, x\right]\right\}$ with $L_{12}^{b}(x, x)=\sigma_{12} /\left(\mu_{2}-\mu_{1}\right)$, and a constraint of continuity: $L_{11}^{a}(x, y)=L_{11}^{b}(x, y)$ along the line $y=\left(\mu_{2} / \mu_{1}\right) x$. For this case, the approximate polynomial Kernel solutions are depicted in Figure 4, for a polynomial degree $d=20$ and maximum error of $3 \cdot 10^{-5}$ with respect to its closed-form

Fig. 4: Approximate solution of the problem (117)-(119) via convex optimization. Domain partitioned as $\Omega_{L}=\Omega_{a} \cup \Omega_{b}$. (a) Kernels $L_{11}^{a}$ and $L_{11}^{b}$. (b) Kernels $L_{12}^{a}$ and $L_{12}^{b}$.

solution [66] (the zero value for the Kernels $L_{11}^{a}$ and $L_{12}^{a}$ are the results of the optimization problem).

As far as the problem (120)-(122) is concerned, for the function $l=l(y)$ considered in [66], its resulting Kernels are continuous and oscillatory (regular Bessel functions), in particular with strong fluctuations as $(x-y) \rightarrow 0$. Under these type of characteristics, the approach proposed is limited by the high polynomial degree required to achieve a precise approximation of oscillatory functions (for example, polynomial approximations of regular Bessel functions require a degree $d \approx 30$. For bivariate polynomials this implies $z(30)=\left(\begin{array}{c}2+30 \\ 30\end{array}\right)=496$ polynomial terms, which is computationally tractable). Instead of using the (arbitrary) function $l=l(y)$ given in [66], to solve the problem (120)-(122) by means of the method proposed, this function has been considered as part of the optimization problem as follows:

$$
\begin{aligned}
& \underset{l=l(y), y \in \Omega}{\operatorname{minimize}} \quad \sigma_{1} \int_{0}^{1} l^{2}(y) d y+\sigma_{2} \int_{0}^{1}\left(\frac{d l}{d y}(y)\right)^{2} d y \\
& \text { subject to: (120)-(122), }
\end{aligned}
$$

with $\sigma_{1} \geq 0$ and $\sigma_{2} \geq 0$ weigh factors to provide tuning in the magnitude and fluctuation of the resulting control action in the motion planning problem. To solve (123)-(124), a convex formulation based on Proposition 2 and Proposition 4 has 
been implemented. This has considered polynomial Kernels of degree $d=28$ to approximate $L_{21}$ and $L_{22}$, and three sets of weigh factors: $\underline{\mathrm{S} 1}: \sigma_{1}=10^{4}, \sigma_{2}=0, \underline{\mathrm{S} 2}: \sigma_{1}=10^{5}$, $\sigma_{2}=10^{4}$ and $\underline{\mathrm{S} 3}: \sigma_{1}=0, \sigma_{2}=10^{4}$. Figure 5 shows the optimal solutions achieved for $l=l(y)=L_{21}(1, y)$ and $L_{22}(1, y)$ in every case. It is worth noting that for the set $\mathrm{S} 1$, the approximate polynomial Kernel solutions correspond to the closed-form solution found in [66].
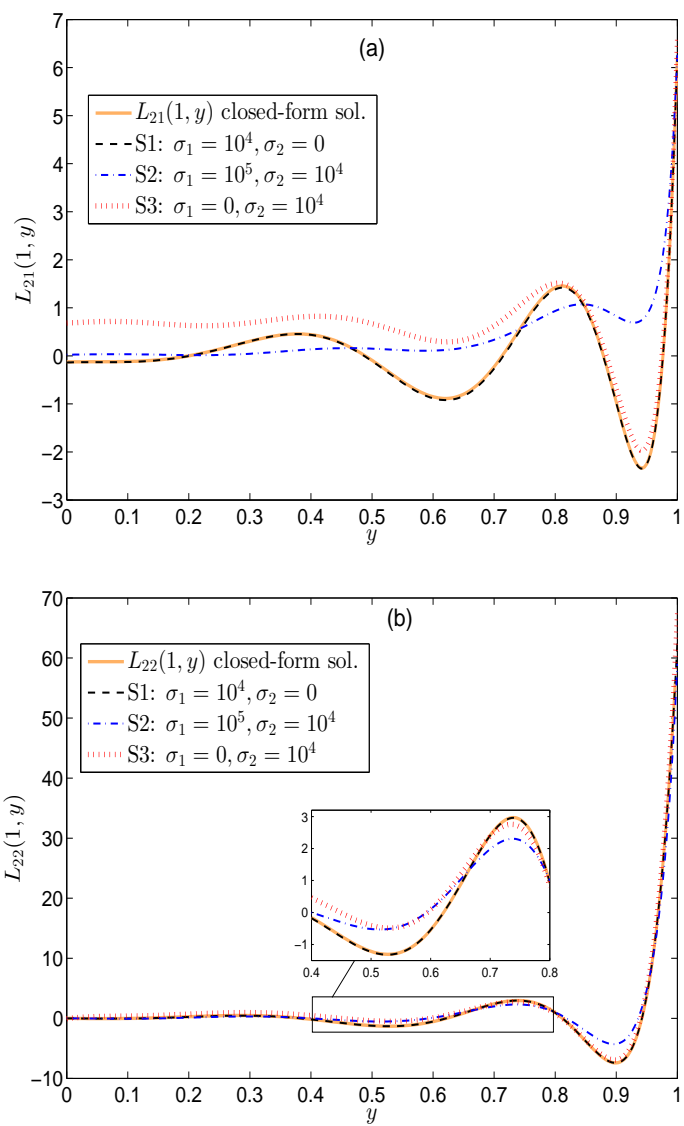

Fig. 5: Approximate solution of the problem (120)-(122), formulated as (123)-(124), via convex optimization. Solutions for the set of weigh factors: S1, S2 and S3. (a) $L_{12}(1, y)$. (b) $L_{22}(1, y)$.

\section{CONCLUSIONS}

In this paper a convex optimization approach to Backstepping PDE design for systems with strict and non-strict feedback structure, involving Volterra and Fredholm operators has been presented. The approach proposed allows obtaining approximate solutions with sufficient precision to guarantee the stability of the system in the $\mathcal{L}^{2}$-norm topology. For polynomial Kernels and continuous functions, uniqueness and invertibility of the Fredholm operator have been proved, which allows applying this methodology to a wide class of problems, without restriction on the spectral characteristic of the resulting approximate operators. The numerical examples illustrate the performance of the approach proposed and the flexibility of SOS-convex optimization to manage problems with operators of different structure and objectives. The method is restricted to systems involving functions which can be approximated by polynomials with computationally tractable degree and Kernels continuous or piece-wise continuous. The main limitation of this method is the current state of Sum-of Squares tools, regarding the type of monomials used in the decompositions, and the convex optimization tools, in relation with managing a large number of parameters and parameters with big magnitudes.

\section{APPENDIX}

Proof of Supporting RESUlts

\section{A. Proof of Lemma 1}

By definition the domains representations in (11) are basic semi-algebraic sets [42]. The equivalence of the sets $\Omega, \Omega_{L}$ and $\Omega_{U}$ and their respective representations in (11) is immediate, following the feasible solution set of the inequalities involved. Moreover, $\Omega$ is a closed and bounded subset of $\mathbb{R}$ as well as the sets $\Omega_{L}$ and $\Omega_{U}$ are in $\mathbb{R}^{2}$, and hence compact [67]. The Archimedean property is verified since the quadratic modules associated to these sets satisfy [51], [48]:

$\exists N \in \mathbb{N}$ s.t.: $N-\sum_{i=1}^{n} x_{i}^{2} \in\left\{s_{0}+\sum_{j=1}^{m} s_{j} g_{j} ;\left(s_{j}\right)_{j=0}^{m} \in \Sigma_{s}\right\}$.

For instance, for $\Omega \equiv\left\{x \in \mathbb{R}, g_{1}(x)=x(1-x) \geq 0\right\}$, selecting $N=1, s_{1}=2 \geq 0$ and $s_{0}=(x-1)^{2} \geq 0$. For the lower triangular domain $\Omega_{L} \equiv\left\{(x, y) \in \mathbb{R}^{2}, g_{1}=x(1-x) \geq\right.$ $\left.0, g_{2}=y(x-y) \geq 0\right\}$, using:

$N_{L}=2 \in \mathbb{N}, s_{1}=4 \geq 0, s_{2}=2 \geq 0$,

$s_{0}=3 x^{2}-2 x y-4 x+y^{2}+2=[1, x, y] Q_{L}[1, x, y]^{T} \in \Sigma_{s}$,

$Q_{L}=\left(\begin{array}{rrr}2 & -2 & 0 \\ -2 & 3 & -1 \\ 0 & -1 & 1\end{array}\right) \succeq 0 \Rightarrow \begin{aligned} & N_{L}-x^{2}-y^{2}= \\ & s_{0}(x, y)+s_{1} g_{1}(x)+s_{2} g_{2}(x, y) .\end{aligned}$

Likewise, in the case of the upper triangular domain $\Omega_{U} \equiv$ $\left\{(x, y) \in \mathbb{R}^{2}, g_{1}(x)=x(1-x) \geq 0, g_{3}(x, y)=(y-x)(1-\right.$ $y) \geq 0\}$, considering:

$N_{U}=3 \in \mathbb{N}, s_{1}=4 \geq 0, s_{2}=2 \geq 0$, $s_{0}=3 x^{2}-2 x-2 y-2 x y+y^{2}+3=[1, x, y] Q_{U}[1, x, y]^{T} \in \Sigma_{s}$,

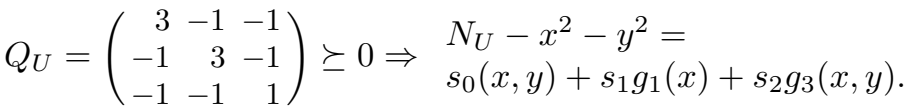

\section{B. Proof of Proposition 1}

Let $V=\frac{1}{2} \int_{0}^{1} w^{2}(x, t) d x=(1 / 2)\|w(x)\|^{2}$ be a Lyapunov functional $^{9}$. Its time-derivative $\dot{V}=\int_{0}^{1} w(x) w_{t}(x) d x$ along the trajectory (15), with $\delta_{0}(x)=0$, is given by:

$$
\begin{aligned}
\dot{V} & =\underbrace{\epsilon \int_{0}^{1} w(x) w_{x x}(x) d x}_{T_{1}}+\underbrace{\int_{0}^{1} w(x) u(x) \delta_{1}(x) d x}_{T_{2}} \\
& -\int_{0}^{1} c(x) w^{2}(x) d x+\underbrace{\int_{0}^{1} w(x) \int_{0}^{x} \delta_{2}(x, y) u(y) d y d x}_{T_{3}} .
\end{aligned}
$$

With respect to the term $T_{1}$, using integration by parts, the boundary conditions in (14), and splitting the resulting expression by a factor $0 \leq \theta \leq 1$, yields:

$$
T_{1}=\left.w(x) w_{x}(x)\right|_{0} ^{1}-\int_{0}^{1} w_{x}^{2}(x) d x,
$$

${ }^{9}$ For a clearer description, the time-dependence in the functions is dropped $(w(x) \equiv w(x, t))$. The norm is the usual in the space of square integrable functions on the domain $\Omega=[0,1]:\|w(x)\|^{2}=\int_{\Omega} w^{2}(x) d x$. 


$$
\leq \underbrace{-\epsilon \theta\left\|w_{x}(x)\right\|^{2}}_{T_{1 a}}-\underbrace{\epsilon(1-\theta)\left\|w_{x}(x)\right\|^{2}}_{T_{1 b}} .
$$

Then, applying the Wirtinger's inequality on the term $T_{1 a}$ and the Agmon's and Young's inequalities on the term $T_{1 b}$ [1], [2], [68], [69], [70], yields:

$$
T_{1} \leq-\epsilon \theta \frac{\pi^{2}}{4}\|w(x)\|^{2}+\epsilon(1-\theta)\left(\|w(x)\|^{2}-\bar{w}^{2}\right),
$$

where $\bar{w}^{2}=\max _{x \in \Omega} w^{2}(x)$, which leads to:

$\dot{V} \leq-\left(\epsilon \theta\left(\frac{\pi^{2}}{2}+2\right)+2(\underline{c}-\epsilon)\right) V(t)-\epsilon(1-\theta) \bar{w}^{2}+T_{2}+T_{3}$.

(128)

with $\underline{c}=\min _{x \in \Omega} c(x)$. As for the term $T_{2}$, substituting $u=$ $u(x, t)$ from (18), the inverse Kernel $L$ from (19), taking an upper bound by means of the maximum absolute value of some integrand functions and the maximum value of the residual functions, yields:

$T_{2} \leq \int_{0}^{1} w^{2}(x)\left|\delta_{1}(x)\right| d x+\int_{0}^{1}|w(x)|\left|\delta_{1}(x)\right| \int_{0}^{x}|L(x, y)||w(y)| d y d x$ $\leq \overline{\delta_{1}}\|w(x)\|^{2}+\bar{\delta}_{1} \bar{w}^{2} \int_{0}^{x} \breve{L}(x, y) d y d x+\sigma \overline{\delta_{1}} \underbrace{\int_{0}^{1}|w(x)| \int_{0}^{x}|w(y)| d y d x}_{T_{2 a}}$,

where $\overline{\delta_{1}}=\max _{x \in \Omega}\left|\delta_{1}(x)\right|$. Then, using the identity $\int_{a}^{b} f(x) \int_{a}^{x} f(y) d y d x=(1 / 2)\left(\int_{a}^{b} f(x) d x\right)^{2}$ for $f$ continuous function [58] on the term $T_{2 a}$, and the Grüss' Integral inequality [71] on its resulting term, leads to:

$$
T_{2} \leq(2+\sigma) \overline{\delta_{1}} V(t)+\overline{\delta_{1}}\left(\frac{\sigma}{8}+m_{0,0}\right) \bar{w}^{2} .
$$

Changing the order of integration in the term $T_{3}$ and following a similar procedure as described above for the term $T_{2}$, yields:

$$
\begin{aligned}
T_{3} & =\int_{0}^{1} u(y) \int_{y}^{1} w(x) \delta_{2}(x, y) d x d y \\
& \leq \int_{0}^{1}|w(x)| \int_{0}^{x}|w(y)|\left|\delta_{2}(x, y)\right| d y d x \\
& +\int_{0}^{1} \int_{0}^{y} \breve{L}(y, s)|w(s)| d s \int_{y}^{1}|w(x)|\left|\delta_{2}(x, y)\right| d x d y \\
& +\sigma \int_{0}^{1} \int_{0}^{y}|w(s)| d s \int_{y}^{1}|w(x)|\left|\delta_{2}(x, y)\right| d x d y \\
& \leq(1+2 \sigma) \overline{\delta_{2}} V(t)+\overline{\delta_{2}}\left(\frac{1+2 \sigma}{8}+m_{0,0}\right) \bar{w}^{2},
\end{aligned}
$$

with $\overline{\delta_{2}}=\max _{(x, y) \in \Omega_{L}}\left|\delta_{2}(x, y)\right|$. Finally, using in (128) the upper bounds (129) and (130) for the terms $T_{2}$ and $T_{3}$, respectively, grouping terms with respect to $V=\|w(x)\|^{2} / 2$ and $\bar{w}^{2}$, the condition $\dot{V} \leq 0$ is satisfied if:

$$
-\left(\overline{\delta_{1}}+\overline{\delta_{2}}\right)+\frac{\overline{\delta_{1}} \sigma+\overline{\delta_{2}}}{2(1+\sigma)}+\frac{\epsilon \theta\left(\pi^{2} / 4+1\right)+(\underline{c}-\epsilon)}{(1+\sigma)} \geq 0
$$

$$
-\left(\overline{\delta_{1}}+\overline{\delta_{2}}\right)+\frac{\overline{\delta_{1}}(1+\sigma)}{\left(8 m_{0,0}+1+2 \sigma\right)}+\frac{\epsilon(1-\theta)}{\left(m_{0,0}+\frac{\sigma+1 / 2}{4}\right)} \geq 0
$$

which leads to the expression (21), i.e. a sufficient condition for exponential stability of (15) in the $\mathcal{L}^{2}$-norm topology.

\section{Proof of Proposition 5}

Let $V=\frac{1}{2} \int_{0}^{1} e^{\alpha x} w^{2}(x, t) d x$ be a Lyapunov functional for some $\alpha>0$. Its time-derivative $\dot{V}=\int_{0}^{1} e^{\alpha x} w(x) w_{t}(x) d x$ along the trajectory (69), with $\delta_{0}(x)=0$ and $\delta_{3}(x)=0$, is given by:

$$
\begin{aligned}
\dot{V} \leq & \underbrace{\int_{0}^{1} e^{\alpha x} w(x) w_{x}(x) d x}_{T_{1}}+\underbrace{\int_{0}^{1} e^{\alpha x} w(x) \int_{0}^{x} u(y) \delta_{1}(x, y) d y d x}_{T_{2}} \\
& \underbrace{+\int_{0}^{1} e^{\alpha x} w(x) \int_{x}^{1} u(y) \delta_{2}(x, y) d y d x}_{T_{3}} .
\end{aligned}
$$

Using integration by parts and the boundary condition in (68) on the term $T_{1}$ yields:

$$
T_{1} \leq\left. e^{\alpha x} w^{2}(x)\right|_{x=0} ^{x=1}-\alpha / 2 \int_{0}^{1} e^{\alpha x} w^{2}(x) d x \leq-\alpha V(t) .
$$

Regarding the term $T_{2}$, changing the order of integration and plugging in $u=u(y, t)$ the expression given by the inverse transformation (88), this can be written as:

$$
\begin{aligned}
T_{2}= & \underbrace{\int_{0}^{1} w(y) \int_{y}^{1} e^{\alpha x} w(x) \delta_{1}(x, y) d x d y}_{T_{2 a}} \\
& +\underbrace{\int_{0}^{1}\left(\int_{0}^{y} R(y, s) w(s) d s\right)\left(\int_{y}^{1} e^{\alpha x} w(x) \delta_{1}(x, y) d x\right) d y}_{T_{2 b}} \\
& +\underbrace{\int_{0}^{1}\left(\int_{y}^{1} S(y, s) w(s) d s\right)\left(\int_{y}^{1} e^{\alpha x} w(x) \delta_{1}(x, y) d x\right) d y}_{T_{2 c}} .
\end{aligned}
$$

Using the Cauchy-Schwarz's integral inequality [72], yields:

$$
\begin{aligned}
T_{2 a}^{2} \leq & \left(\int_{0}^{1} e^{2 \alpha x} w^{2}(x) d x\right)\left(\int_{0}^{1}\left(\int_{0}^{x} w(y) \delta_{1}(x, y) d y\right)^{2} d x\right) \\
\leq & \max _{x \in \Omega}\left\{e^{\alpha x}\right\} \max _{y \in \Omega}\left\{e^{-\alpha y}\right\} 4 V^{2}(t) \cdot \\
& \left(\int_{0}^{1}\left(\int_{0}^{x} e^{\alpha y} w^{2}(y) d y\right)\left(\int_{0}^{x} \delta_{1}^{2}(x, y) d y\right) d x\right) \Rightarrow \\
T_{2 a} \leq & 2 e^{\alpha / 2} V(t) \sqrt{\Delta_{1}}, \\
T_{2 b}^{2} \leq & \left(\int_{0}^{1}\left(\int_{0}^{y} R(y, s) w(s) d s\right)^{2} d y\right)\left(\int_{0}^{1}\left(\int_{y}^{1} e^{\alpha x} w(x) \delta_{1}(x, y) d x\right)^{2} d y\right) \\
\leq & \max _{x \in \Omega}\left\{e^{\alpha x}\right\} \max _{s \in \Omega}\left\{e^{-\alpha s}\right\}\left(\int_{0}^{1}\left(\int_{0}^{y} R^{2}(y, s) d s\right) .\right. \\
& \left.\left(\int_{0}^{y} e^{\alpha s} w^{2}(s) d s\right) d y\right)\left(\int_{0}^{1}\left(\int_{y}^{1} e^{\alpha x} w^{2}(x) d x\right) .\right. \\
& \left.\left(\int_{y}^{1} \delta_{1}^{2}(x, y) d x\right) d y\right) \Rightarrow \\
T_{2 b} \leq & 2 e^{\alpha / 2} V(t) \sqrt{\Delta_{1}}\left(\int_{0}^{1} \int_{0}^{y} R^{2}(y, s) d s d y\right)^{\frac{1}{2}},
\end{aligned}
$$

and
$T_{2 c}^{2} \leq\left(\int_{0}^{1}\left(\int_{y}^{1} S(y, s) w(s) d s\right)^{2} d y\right)\left(\int_{0}^{1}\left(\int_{y}^{1} e^{\alpha x} w(x) \delta_{1}(x, y) d x\right)^{2} d y\right)$ $\leq 4 e^{\alpha} V^{2}(t)\left(\int_{0}^{1}\left(\int_{y}^{1} S^{2}(y, s) d s\right) d y\right)\left(\int_{0}^{1}\left(\int_{y}^{1} \delta_{1}^{2}(x, y) d x\right) d y\right) \Rightarrow$ $T_{2 c} \leq 2 e^{\alpha / 2} V(t) \sqrt{\Delta_{1}}\left(\int_{0}^{1} \int_{y}^{1} S^{2}(y, s) d s d y\right)^{\frac{1}{2}}$,

where $\Delta_{1}=\int_{0}^{1} \int_{0}^{x} \delta_{1}^{2}(x, y) d y d x$. With respect to the term $T_{3}$, this can be written as:

$$
T_{3}=\underbrace{\int_{0}^{1} w(y) \int_{0}^{y} e^{\alpha x} w(x) \delta_{2}(x, y) d x d y}_{T_{3 a}}
$$




$$
\begin{aligned}
& +\underbrace{\int_{0}^{1}\left(\int_{0}^{y} R(y, s) w(s) d s\right)\left(\int_{0}^{y} e^{\alpha x} w(x) \delta_{2}(x, y) d x\right) d y}_{T_{3 b}} \\
& +\underbrace{\int_{0}^{1}\left(\int_{y}^{1} S(y, s) w(s) d s\right)\left(\int_{0}^{y} e^{\alpha x} w(x) \delta_{2}(x, y) d x\right) d y}_{T_{3 c}},
\end{aligned}
$$

where upper bounds for every term, in relation with $\Delta_{2}=$ $\int_{0}^{1} \int_{x}^{1} \delta_{2}^{2}(x, y) d y d x$, can be found following the same procedure described above in (136)-(138). Finally, using in (133) the upper bounds for the terms $T_{1}, T_{2}$ and $T_{3}$, the condition $\dot{V} \leq 0$ is satisfied provided:

$$
\alpha-2 e^{\alpha / 2}\left(\sqrt{\Delta_{1}}+\sqrt{\Delta_{2}}\right)\left(1+\sqrt{\sigma_{1}}+\sqrt{\sigma_{2}}\right) \geq 0,
$$

with $\sigma_{1}=\int_{0}^{1} \int_{0}^{x} R^{2}(x, y) d y d x$ and $\sigma_{2}=\int_{0}^{1} \int_{x}^{1} S^{2}(x, y) d y d x$. Since the factor $(1 / 2) \alpha e^{-\alpha / 2}$ reaches the maximum value of $e^{-1}$ at $\alpha=2$, the expression (89) is obtained, i.e. a sufficient condition for exponential stability of $(69)$ in the $\mathcal{L}^{2}$-norm topology.

\section{REFERENCES}

[1] M. Krstic and A. Smyshlyaev, Boundary Control of PDEs: A Course on Backstepping Designs. SIAM, 2008.

[2] A. Smyshlyaev and M. Krstic, Adaptive Control of Parabolic PDEs. Princeton University Press, 2010.

[3] R. Vazquez and M. Krstic, Control of Turbulent and Magnetohydrodynamic Channel Flows. Boundary Stabilization and State Estimation. Birkhäuser, 2007.

[4] W. J. Liu, Elementary Feedback Stabilization of the Linear ReactionConvection-Diffusion Equation and the Wave Equation. Springer, 2009.

[5] T. Meurer, Control of Higher-Dimensional PDEs: Flatness and Backstepping Designs. Springer, 2013.

[6] F. Riesz and B. Sz.-Nagy, Functional Analysis. Dover Publications, 1990.

[7] S. M. Zemyan, The Classical Theory of Integral Equations: A Concise Treatment. Birkhäuser, 2012.

[8] D. Colton, "The Solution of Initial-Boundary Value Problems for Parabolic Equations by the Method of Integral Operators," Journal of Differential Equations, vol. 26, no. 2, pp. 181-190, 1977.

[9] T. I. Seidman, "Two results on exact boundary control of parabolic equations," Journal Applied Mathematics and Optimization, vol. 11, no. 1, pp. 145-152, 1984.

[10] W. Harmon Ray, Advanced Process Control. McGraw-Hill, 1981.

[11] P. Christofides, Nonlinear and Robust Control of PDE Systems: Methods and Applications to Transport-Reaction Processes. Birkhäuser, 2001.

[12] R. F. Curtain and H. J. Zwart, An Introduction to Infinite Dimensional Linear Systems Theory. Springer-Verlag, 1995.

[13] A. Bensoussan, G. Da Prato, M.C. Delfour and S.K Mitter, Representation and Control of Infinite Dimensional Systems. 2nd edition. Birkhäuser, 2007.

[14] M. Tucsnak and G. Weiss, Observation and Control for Operator Semigroups. Birkhäuser, 2009.

[15] W. J. Liu, "Boundary feedback stabilization of an unstable heat equation," SIAM Journal on Control and Optimization, vol. 42, no. 3, pp. 1033-1043, 2003.

[16] I. Gohberg, S. Goldbergand and M. Kaashoek, Basic Classes of Linear Operators. Birkhäuser, 2003.

[17] C. Corduneanu, Functional Equations with Causal Operators. CRC Press, Taylor \& Francis, 2002.

[18] A. Bensoussan, M. C. Delfour, and S. K. Mitter, "The linear quadratic optimal control problem for infinite dimensional systems over an infinite horizon; survey and examples," in Proc. Conf. on Decision and Control, 1976, pp. 746-751.

[19] A. Smyshlyaev and M. Krstic, "Closed-Form Boundary State Feedbacks for a Class of 1-D Partial Integro-Differential Equations," IEEE Transactions on Automatic Control, vol. 49, no. 12, pp. 2185-2202, 2004
[20] M. Krstic and A. Smyshlyaev, "Backstepping boundary control for first-order hyperbolic PDEs and application to systems with actuator and sensor delays," Systems \& Control Letters, vol. 57, issue 9, pp. 750-758, 2008.

[21] A. Jerri, Introduction to Integral Equations with Applications. 2nd edition. Wiley, 1999.

[22] M. A. Khamsi and W. A. Kirk, An Introduction to Metric Spaces and Fixed Point Theory. John Wiley \& Sons, 2001.

[23] R. Kress, Linear Integral Equations. 3rd edition. Springer, 2014.

[24] F. Cali, E. Marchetti and V. Muresan, "On some Volterra-Fredholm Integral Equation,". International Journal of Pure and Applied Mathematics, vol. 31, no. 2, pp. 173-184, 2006.

[25] C. Guo, C. Xie and C. Zhou, "Stabilization of a spatially non-causal reaction-diffusion equation by boundary control," International Journal of Robust and Nonlinear Control, vol. 24, no. 1, pp. 1-17, 2012.

[26] D. Tsubakino, F. Bribiesca-Argomedo and M. Krstic, "BacksteppingForwarding Control of Parabolic PDEs with Partially Separable Kernels," in Proc. Conf. on Decision and Control, 2014, pp. 5236-5241.

[27] F. Bribiesca-Argomedo and M. Krstic, "Backstepping-Forwarding Boundary Control Design for First-Order Hyperbolic Systems With Fredholm Integrals," in Proc. American Control Conference, 2014, pp. 5428-5433.

[28] F. Bribiesca-Argomedo and M. Krstic, "Backstepping-Forwarding Control and Observation for Hyperbolic PDEs with Fredholm Integrals," IEEE Transactions on Automatic Control, vol. 60, no. 8, pp. 2145-2160, 2015.

[29] N. Bekiaris-Liberis and M. Krstic, "Lyapunov Stability of Linear Predictor Feedback for Distributed Input Delays," IEEE Transactions on Automatic Control, vol. 56, no. 3, pp. 655-660, 2011.

[30] R. Vazquez, "Boundary Control Laws and Observer Design for Convective, Turbulent and Magnetohydrodynamic Flows," PhD Thesis, University of California, San Diego, 2006.

[31] D. Tsubakinoa and S. Harab, "Backstepping observer design for parabolic PDEs with measurement of weighted spatial averages," Automatica, vol. 53, pp. 179-187, 2015.

[32] S. Almezel, Q. H. Ansari and M. A. Khamsi (Editors), Topics in Fixed Point Theory. Springer, 2014.

[33] P. Ascencio, A. Astolfi and T. Parisini, "Backstepping PDE Design, Volterra and Fredholm Operators: a Convex Optimization Approach," in Proc. IEEE Conf. on Decision and Control, 2015, pp. 7048-7053.

[34] P. Ascencio, A. Astolfi and T. Parisini, "An Adaptive Observer for a class of Parabolic PDEs based on a Convex Optimization Approach of Backstepping PDE Design," in Proc. American Control Conference, 2016, pp. 3429-3434.

[35] P. Ascencio, A. Astolfi and T. Parisini, "Backstepping PDE-Based Adaptive Observer for a Single Particle Model of Lithium-Ion Batteries." Accepted in $55^{\text {th }}$ IEEE Conf. on Decision and Control, USA, 2016.

[36] P. R. Halmos and V. S. Sunder. Bounded integral operators on $L_{2}$ spaces. Springer-Verlag, 1978

[37] B. Rynne and M. Youngson, Linear Functional Analysis. 2nd edition. Springer, 2008.

[38] A. Bowers, N.J. Kalton, An Introductory Course in Functional Analysis. Springer, 2014.

[39] A.N. Kolmogorov and S.V. Fomin, Elements of the Theory of Functions and Functional Analysis. Volume 1: Metric and Normed Spaces. Graylock Press, 1957.

[40] K. Atkinson and W. Han, Theoretical Numerical Analysis: A Functional Analysis Framework. 3rd edition. Springer, 2009.

[41] R. A. Kennedy and P. Sadeghi, Hilbert Space Methods in Signal Processing. Cambridge University Press, 2013.

[42] G. Blekherman, P. Parrilo and R. Thomas, Semidefinite Optimization and Convex Algebraic Geometry. SIAM, 2013.

[43] Reza Kamyar and Matthew M. Peet, "Polynomial Optimization with Applications to Stability Analysis and Control - Alternatives to Sum of Squares," Journal of Discrete and Continuous Dynamical Systems Series B, vol. 20, no. 8, pp. 2383-2417, 2015.

[44] P. Parrilo, "Structured semidefinite programs and semialgebraic geometry methods in robustness and optimization," $\mathrm{PhD}$ thesis, California Institute of Technology, 2000.

[45] P. Parrilo, "Semidefinite programming relaxations for semialgebraic problems," Mathematical Programming, Ser. B 96, pp. 293-320, 2003.

[46] J.B. Lasserre, "Global optimization with polynomials and the problem of moments," SIAM Journal on Optimization, vol. 11, no. 3, pp. 796817,2001

[47] J. B. Lasserre, Moments, Positive Polynomials and Their Applications. Imperial College Press, 2010. 
[48] J. B. Lasserre, An Introduction to Polynomial and Semi-Algebraic Optimization. Cambridge University Press, 2015.

[49] K. Schmüdgen, "The K-moment problem for compact semi-algebraic sets," Mathematische Annale, vol. 289, no.1, pp. 203-206, 1991.

[50] M. Putinar, "Positive polynomials on compact semi-algebraic sets," Indiana University Mathematics Journal, vol. 42, pp. 969-984, 1993.

[51] M. Laurent, "Sums of squares, moment matrices and optimization over polynomials," Springer, Emerging Applications of Algebraic Geometry, IMA Volumes in Mathematics and its Applications, vol. 149, pp. 157270, 2009. [Online]. Available: http://homepages.cwi.nl/ monique/

[52] Friedrich Sauvigny. "Partial Differential Equations: Vol. 1 Foundations and Integral Representations". Springer, 2006.

[53] Dzung Minh Ha. "Functional Analysis. Volume I: A Gentle Introduction". Matrix Editions, 2006.

[54] Michael J. D. Powell. "Approximation Theory and Methods". Cambridge University Press, 1981.

[55] C.W. Scherer and C.W.J. Hol, "Matrix Sum-of-Squares Relaxations for Robust Semi-Definite Programs," Mathematical Programming, vol. 107, no. 1-2, pp.189-211, 2006

[56] S.Krantz and H. Parks, A Primer of Real Analytic Functions. 2nd edition. Birkhäuser, 2002

[57] C. Meyer, Matrix Analysis and Applied Linear Algebra. SIAM, 2000.

[58] P. M. Fitzpatrick, Advanced Calculus. 2nd edition. American Mathematical Society, 2009.

[59] H. Garth Dales, P. Aiena, J. Eschmeier, K. Laursen and G. Willis "Introduction to Banach Algebras, Operators, and Harmonic Analysis". Cambridge University Press, 2003.

[60] V. S. Sunder. "Functional Analysis: Spectral Theory". Birkhäuser Advanced Texts, 1998.

[61] C.S. Kubrusly, The Elements of Operator Theory. 2nd edition. Birkhäuser, 2010.

[62] A. T. Bharucha-Reid, Random Integral Equations. Academic Press, 1972.

[63] E. Davies, Linear Operators and their Spectra. Cambridge University Press, 2007.

[64] J. Löfberg. "YALMIP : A Toolbox for Modeling and Optimization in MATLAB," in Proc. CACSD Conference, 2004. [Online]. Available: http://users.isy.liu.se/johanl/yalmip

[65] The MOSEK optimization toolbox for MATLAB, version 8. Denmark: MOSEK ApS. [Online]. Available: https://www.mosek.com/

[66] L. Hu, F. Di Meglio, R. Vazquez, and M. Krstic,"Control of Homodirectional and General Heterodirectional Linear Coupled Hyperbolic PDEs," IEEE Transactions on Automatic Control, vol. 61, no. 10, pp. 3301-3314, 2016.

[67] W. A. Sutherland, Introduction to Metric and Topological Spaces. 2nd edition. Oxford Universiy Press, 1981.

[68] G. H. Hardy, J. E. Littlewood and G. Polya, Inequalities. 2nd edition. Cambridge University Press, 1952.

[69] D. Boyd, "Best constants in a class of integral inequalities," Pacific Journal of Mathematics, vol. 30, no. 2, pp. 367-383, 1969.

[70] T. X. Wang, "Stability in Abstract Functional Differential Equations. Part II. Applications," Journal of Mathematical Analysis and Applications, vol. 186, issue 3, pp. 835-861, 1994.

[71] S.S. Dragomir, "Some Integral Inequalities of Grüss type," RGMIA Research Report Collection, Vol. 1, no. 2, pp. 95-111, 1998.

[72] J. Michael Steele, The Cauchy Schwarz Master Class. An Introduction to the Art of Mathematical Inequalities. Cambridge University Press, 2004

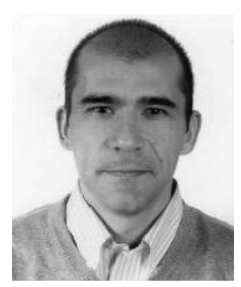

Pedro Ascencio was born in Concepción, Chile, in 1973. He received the B.Sc. and M.Sc. degrees in Electrical Engineering from the University of Concepción, Chile, in 1998 and 2001, respectively. From 2001 to 2013, he was Control Systems Engineer working on industrial processes automation. $\mathrm{He}$ received the PhD. degree in Control Systems in 2017 from Imperial College London. His research interests are focussed on State and Parameter Estimation for Finite and Infinite Dimensional Systems.

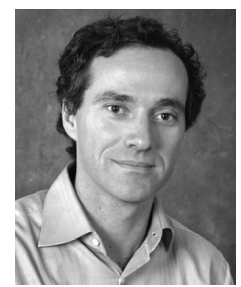

Alessandro Astolfi (F'09) was born in Rome, Italy, in 1967. He graduated in electrical engineering from the University of Rome in 1991. In 1992 he joined ETH-Zurich where he obtained a M.Sc. in Information Theory in 1995 and the Ph.D. degree with Medal of Honor in 1995 with a thesis on discontinuous stabilization of non-holonomic systems. In 1996 he was awarded a Ph.D. from the University of Rome "La Sapienza" for his work on nonlinear robust control. Since 1996 he has been with the Electrical and Electronic Engineering Department of Imperial College London, London (UK), where he is currently Professor in Nonlinear Control Theory and Head of the Control and Power Group. From 1998 to 2003 he was also an Associate Professor at the Dept. of Electronics and Information of the Politecnico of Milano. Since 2005 he has also been a Professor at Dipartimento di Ingegneria Civile e Ingegneria Informatica, University of Rome "Tor Vergata". He has been a visiting lecturer in "Nonlinear Control" in several universities, including ETH-Zurich (1995-1996); Terza University of Rome (1996); Rice University, Houston (1999); Kepler University, Linz (2000); SUPELEC, Paris (2001). His research interests are focused on mathematical control theory and control applications, with special emphasis for the problems of discontinuous stabilization, robust and adaptive control, observer design and model reduction. He is the author of more than 120 journal papers, of 30 book chapters and of over 240 papers in refereed conference proceedings. He is the recipient of the IEEE CSS A. Ruberti Young Researcher Prize (2007) and of the IEEE CSS George S. Axelby Outstanding Paper Award (2012). He is a "Distinguished Member" of the IEEE CSS. He is the author (with D. Karagiannis and R. Ortega) of the monograph "Nonlinear and Adaptive Control with Applications" (Springer-Verlag). He is Associate Editor of Automatica, the International Journal of Control, the Journal of the Franklin Institute, and the International Journal of Adaptive Control and Signal Processing. He is Senior Editor of the IEeE Transactions On Automatic CONTROL and Editor-in-Chief of the European Journal of Control. He has also served in the IPC of various international conferences. He is currently the Chair of the IEEE CSS Conference Editorial Board.

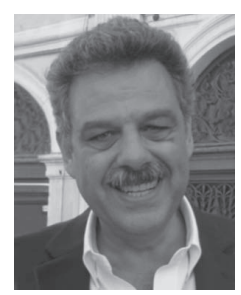

Thomas Parisini (F'11) received the Ph.D. degree in Electronic Engineering and Computer Science in 1993 from the University of Genoa. He was with Politecnico di Milano and since 2010 he holds the Chair of Industrial Control and is Director of Research at Imperial College London. He is a Deputy Director of the KIOS Research and Innovation Centre of Excellence, University of Cyprus. Since 2001 he is also Danieli Endowed Chair of Automation Engineering with University of Trieste. In 20092012 he was Deputy Rector of University of Trieste. He authored or co-authored more than 270 research papers in archival journals, book chapters, and international conference proceedings. His research interests include neural-network approximations for optimal control problems, fault diagnosis for nonlinear and distributed systems, nonlinear model predictive control systems and nonlinear estimation. He is a co-recipient of the IFAC Best Application Paper Prize of the Journal of Process Control, Elsevier, for the three-year period 2011-2013 and of the 2004 Outstanding Paper Award of the IeEe Transactions On Neural Networks. He is also a recipient of the 2007 IEEE Distinguished Member Award. In 2016 he was awarded as Principal Investigator at Imperial of the H2020 European Union flagship Teaming Project KIOS Research and Innovation Centre of Excellence led by University of Cyprus. In 2012 he was awarded an ABB Research Grant dealing with energy-autonomous sensor networks for selfmonitoring industrial environments. Thomas Parisini currently serves as VicePresident for Publications Activities of the IEEE Control Systems Society and during 2009-2016 he was the Editor-in-Chief of the IEEE TransaCtions ON Control Systems Technology. Since 2017, he is Editor for Control Applications of Automatica. He is also the Chair of the IFAC Technical Committee on Fault Detection, Supervision \& Safety of Technical Processes - SAFEPROCESS. He was the Chair of the IEEE Control Systems Society Conference Editorial Board and a Distinguished Lecturer of the IEEE Control Systems Society. He was an elected member of the Board of Governors of the IEEE Control Systems Society and of the European Control Association (EUCA) and a member of the board of evaluators of the 7th Framework ICT Research Program of the European Union. Prof. Parisini is currently serving as an Associate Editor of the Int. J. of Control and served as Associate Editor of the IEEe Transactions On Automatic Control, of the IEEE Transactions On Neural Networks, of Automatica, and of the Int. J. of Robust and Nonlinear Control. 